\title{
Particles and fields in superfluids: Insights from the two-dimensional Gross-Pitaevskii equation
}

\author{
Vishwanath Shukla, ${ }^{1, *}$ Rahul Pandit, ${ }^{2, \dagger}$ and Marc Brachet ${ }^{3, \dagger}$ \\ ${ }^{1}$ Service de Physique de l'État Condensé, Université Paris-Saclay, CEA Saclay, 91191 Gif-sur-Yvette, France \\ ${ }^{2}$ Centre for Condensed Matter Theory, Department of Physics, Indian Institute of Science, Bangalore 560012, India \\ ${ }^{3}$ Laboratoire de Physique Statistique de l'Ecole Normale Supérieure, associé au CNRS et aux Universités Paris VI et VII, \\ 24 Rue Lhomond, 75231 Paris, France
}

(Received 26 October 2017; published 24 January 2018)

\begin{abstract}
We carry out extensive direct numerical simulations to investigate the interaction of active particles and fields in the two-dimensional Gross-Pitaevskii superfluid, in both simple and turbulent flows. The particles are active in the sense that they affect the superfluid even as they are affected by it. We tune the mass of the particles, which is an important control parameter. At the one-particle level, we show how light, neutral, and heavy particles move in the superfluid, when a constant external force acts on them; in particular, beyond a critical velocity, at which a vortex-antivortex pair is emitted, particle motion can be periodic or chaotic. We demonstrate that the interaction of a particle with vortices leads to dynamics that depends sensitively on the particle characteristics. We also demonstrate that assemblies of particles and vortices can have rich, and often turbulent, spatiotemporal evolution. In particular, we consider the dynamics of the following illustrative initial configurations: (a) one particle placed in front of a translating vortex-antivortex pair; (b) two particles placed in front of a translating vortex-antivortex pair; (c) a single particle moving in the presence of counter-rotating vortex clusters; (d) four particles in the presence of counter-rotating vortex clusters. We compare our work with earlier studies and examine its implications for recent experimental studies in superfluid helium and Bose-Einstein condensates.
\end{abstract}

DOI: 10.1103/PhysRevA.97.013627

\section{INTRODUCTION}

The study of the dynamics of particles immersed in a superfluid has a long history [1]. This venerable subject has experienced a renaissance because of recent experiments that (a) use particles in superfluid helium [2-6] to track vortex lines or (b) to study impurities in cold-atom, Bose-Einstein condensates (BECs) [7].

Unlike particles moving through a viscous fluid, particles that move with a constant speed through a superfluid move without resistance, so long as this speed lies below a critical threshold [8-12]. Above this threshold, superfluidity breaks down. This critical speed has been related, in the GrossPitaevskii equation (GPE), to vortex generation, which is caused by a saddle-node bifurcation of steady states $[8,9,11-$ 13]. At supercritical speeds, vortex generation is associated with forces that act on particles. The interaction of such vortices with single particles or assemblies of particles are problems of central importance in this rapidly developing field. The motion of a single particle, which is affected by the superflow and acts on it too, has been studied in Refs. [12-14] in a Gross-Pitaevskii (GP) superfluid. We refer to this as an active particle.

To go beyond earlier theoretical and numerical studies [13-15] of this problem, we have developed a minimal model $[16,17]$ recently. In this model, the equations of motion of particles are coupled with the GP field $\psi$, which is used often

\footnotetext{
*research.vishwanath@gmail.com

${ }^{\dagger}$ Also at Jawaharlal Nehru Centre For Advanced Scientific Research, Jakkur, Bangalore, India; rahul@iisc.ac.in

†brachet@physique.ens.fr
}

to describe a weakly interacting superfluid at low temperatures. Furthermore, our model includes a particle-particle, short-range repulsive force. This model generates naturally an effective superfluid-mediated attractive interaction between the particles; we have shown $[16,17]$ that the interplay between the short-range particle repulsion and the superfluid-mediated attraction leads to a sticking transition at which the coefficient of restitution, for two-particle collisions, vanishes.

We build on our study in Ref. [16] and consider the interaction of particles with vortices. We begin with a brief, qualitative overview of our principal results, which we obtain from extensive direct numerical simulations (DNSs) of the interaction of particles and fields in the two-dimensional (2D) GPE, in both simple and turbulent flows. At the one-particle level, we explore, for light, neutral, and heavy particles, the nature of their dynamics in the superfluid, when a constant external force acts on them; in particular, we show, by a careful consideration of the effects of the particle mass, how the motion of such particles can be temporally periodic or chaotic. We demonstrate that the interaction of a particle with vortices leads to dynamics that depends sensitively on the particle characteristics. For assemblies of particles and vortices we demonstrate that their dynamics show rich, turbulent spatiotemporal evolution. In particular, we systematize the spatiotemporal evolution of an initial configuration in which one particle is placed in front of a translating vortex-antivortex pair. We then examine the complicated motions of two particles placed in front of a translating vortex-antivortex pair and show that, when a vortex traps a particle, we can use this particle as a tracer that can track vortex motion. As an illustrative example, we first examine the motion of a single particle in 
the presence of many vortices in a decaying turbulent flow. We then study how the interactions of many particles with fields in the presence of a group of counter-rotating vortices can lead to turbulent and spatiotemporally chaotic evolution. We compare our work with earlier studies and discuss the experimental implications of our work.

The remainder of this paper is organized as follows. In the first part of Sec. II we recapitulate the essentials of our model [16] for particles coupled to the GP field $\psi$; in a subsection, we present the numerical methods we use in our studies of this model. Section III is devoted to our results for different classes of initial conditions; the first subsection here discusses vortex generation by the motion of a single particle (light, neutral, or heavy) and the spatiotemporal evolution of $\psi$ and the particle position; the second subsection explores the interaction between particles and a vortex-antivortex pair; the third subsection considers illustrative multiparticle and multivortex assemblies and studies their rich and turbulent spatiotemporal evolution. In Sec. IV we give our conclusions and discuss the significance of our results.

\section{MODEL AND NUMERICAL METHODS}

\section{A. Model}

We begin with a brief recapitulation of our minimal model [16] of active and interacting particles in a weakly interacting Bose superfluid at zero temperature. The dynamics of these particles is governed by the Lagrangian

$$
\begin{aligned}
\mathcal{L}= & \int_{\mathcal{A}}\left[\frac{i \hbar}{2}\left(\psi^{*}(\mathbf{r}, t) \frac{\partial \psi(\mathbf{r}, t)}{\partial t}-\psi(\mathbf{r}, t) \frac{\partial \psi^{*}(\mathbf{r}, t)}{\partial t}\right)\right. \\
& -\frac{\hbar^{2}}{2 m} \nabla \psi(\mathbf{r}, t) \cdot \nabla \psi^{*}(\mathbf{r}, t)+\mu|\psi(\mathbf{r}, t)|^{2} \\
& \left.-\frac{g}{2}|\psi(\mathbf{r}, t)|^{4}-\sum_{i=1}^{\mathcal{N}_{0}} V_{\mathcal{P}}\left(\mathbf{r}-\mathbf{q}_{i}\right)|\psi(\mathbf{r}, t)|^{2}\right] d \mathbf{r} \\
& +\frac{m_{o}}{2} \sum_{i=1}^{\mathcal{N}_{0}} \dot{q}_{i}^{2}-\sum_{i, j, i \neq j}^{\mathcal{N}_{0}, \mathcal{N}_{0}} \frac{\Delta_{E} r_{\mathrm{SR}}^{12}}{\left|\mathbf{q}_{i}-\mathbf{q}_{j}\right|^{12}},
\end{aligned}
$$

where $\psi$ is the complex, condensate wave function, $\psi^{*}$ is its complex conjugate, $m$ is the mass of the bosons, $\mathcal{A}$ is the simulation domain, $g$ is the effective interaction strength, $\mu$ is the chemical potential, $V_{\mathcal{P}}$ is the potential that accounts for the particles, $q_{i}$ is the position of particle $i$, and $\mathcal{N}_{0}$ is the total number of particles, each with mass $m_{o}$. The last term in Eq. (1) is the short-range (SR) repulsive, two-particle potential, which is characterized by the parameters $\Delta_{E}$ and $r_{\mathrm{SR}}$.

From the Lagrangian (1) we obtain the following GP equation for the field $\psi$ :

$$
i \hbar \frac{\partial \psi}{\partial t}=-\frac{\hbar^{2}}{2 m} \nabla^{2} \psi-\mu \psi+g|\psi|^{2} \psi+\sum_{i=1}^{\mathcal{N}_{0}} V_{\mathcal{P}}\left(\mathbf{r}-\mathbf{q}_{i}\right) \psi
$$

and, for the particle $i$, we get

$$
m_{o} \ddot{\mathbf{q}}_{i}=\mathbf{f}_{o, i}+\mathbf{f}_{\mathrm{SR}, i},
$$

where

$$
\mathbf{f}_{o, i}=\int_{\mathcal{A}}|\psi|^{2} \nabla V_{\mathcal{P}} d \mathbf{r}
$$

and $\mathbf{f}_{\mathrm{SR}, i}$ arises because of the SR potential. If there is no external force, the total energy of this system

$$
E=E_{\mathrm{F}}+E_{\mathrm{o}}^{\mathrm{T}}+E_{\mathrm{SR}}
$$

is conserved; here, $E_{\mathrm{F}}$, the energy of the superfluid field, $E_{\mathrm{o}}^{\mathrm{T}}$, the total kinetic energy of the particles, and $E_{\mathrm{SR}}$, the energy from the SR repulsion between the particles, are defined, respectively, as follows:

$$
\begin{aligned}
E_{\mathrm{F}}= & \frac{1}{\mathcal{A}} \int_{\mathcal{A}}\left[\frac{\hbar^{2}}{2 m}|\nabla \psi|^{2}+\frac{1}{2} g\left(|\psi|^{2}-\frac{\mu}{g}\right)^{2}\right. \\
& \left.+\sum_{i=1}^{\mathcal{N}_{\mathrm{o}}} V_{\mathcal{P}}\left(\mathbf{r}-\mathbf{q}_{i}\right)|\psi|^{2}\right] d \mathbf{r}, \\
E_{\mathrm{o}}^{\mathrm{T}}= & \frac{1}{\mathcal{A}} \sum_{i=1} \frac{1}{2} m_{\mathrm{o}} \dot{\mathbf{q}}_{i}^{2}, \\
E_{\mathrm{SR}}= & \frac{1}{\mathcal{A}} \sum_{i, j, i \neq j}^{\mathcal{N}_{\mathrm{o}}, \mathcal{N}_{\mathrm{o}}} \frac{\Delta_{E} r_{\mathrm{SR}}^{12}}{\left|q_{i}-q_{j}\right|^{12}} .
\end{aligned}
$$

The dynamical evolution of Eqs. (2) and (3) conserves the total momentum

$$
\mathbf{P}(t)=\int_{\mathcal{A}} \frac{i \hbar}{2}\left(\psi^{*} \nabla \psi-\psi \nabla \psi^{*}\right) d \mathbf{r}+\sum_{i=1}^{\mathcal{N}_{\mathrm{o}}} m_{\mathrm{o}} \dot{\mathbf{q}}_{i}
$$

and the number of bosons

$$
N=\int_{\mathcal{A}}|\psi|^{2} d \mathbf{r}
$$

We use the Madelung transformation

$$
\psi(\mathbf{r}, t)=\sqrt{\rho(\mathbf{r}, t) / m} \exp [i \phi(\mathbf{r}, t)]
$$

to express the GP equation in terms of hydrodynamical variables; here, $\rho(\mathbf{r}, t)$ and $\phi(\mathbf{r}, t)$ are, respectively, the density and phase fields, and the superfluid velocity is

$$
\mathbf{v}(\mathbf{r}, t)=(\hbar / m) \nabla \phi(\mathbf{r}, t) .
$$

Hence we note that the flow is irrotational in the absence of any quantum vortices.

Linearization of the GP equation for a uniform system around the equilibrium state with constant density $\rho_{0}$ yields the Bogoliubov dispersion relation

$$
\omega(k)=c k\left(1+\frac{1}{2} \xi^{2} k^{2}\right)^{1 / 2},
$$

where $k$ is the wave number; for small wave numbers $k$ the spectrum is soundlike, with sound velocity $c=\sqrt{g \rho_{0} / m^{2}}$; for length scales smaller than $\xi$, i.e., $k \gtrsim 1 / \xi$, dispersive effects are observed. The length scale $\xi=\sqrt{\hbar^{2} / 2 g \rho_{0}}$ is called the healing length scale and is also associated with the vortex core size.

We model the particles by specifying the potential $V_{\mathcal{P}}$, which allows us to choose the shape and size of the particles. In this study, we use the Gaussian potential

$$
V_{\mathcal{P}}=V_{o} \exp \left(-\frac{r^{2}}{2 d_{p}^{2}}\right)
$$


Here $V_{o}$ is the strength of the potential and $d_{\mathrm{p}}$ is the measure of its width. The introduction of a particle displaces some superfluid, with area comparable to that occupied by the particle. The mass of the displaced fluid is given by

$$
m_{\mathrm{f}}=m \int_{\mathcal{A}}\left(\left|\psi_{\text {uniform }}\right|^{2}-\left|\psi_{\text {particle }}\right|^{2}\right) d \mathbf{r},
$$

where $\psi_{\text {uniform }}$ and $\psi_{\text {particle }}$ are the wave functions of the minimum-energy state of the uniform system in the absence of any particle and in the presence of a single particle, respectively. We use the mass $m_{\mathrm{f}}$ of the displaced superfluid to define the ratio

$$
\mathcal{M} \equiv \frac{m_{o}}{m_{\mathrm{f}}}
$$

which allows us to distinguish between (1) heavy $(\mathcal{M}>1)$, (2) neutral $(\mathcal{M}=1)$, and (3) light $(\mathcal{M}<1)$ particles.

\section{B. Numerical methods}

To study the dynamics of particles in complex superfluid flows, we solve Eqs. (2) and (3) numerically. We perform direct numerical simulations (DNSs) of the GPE by using the Fourier pseudospectral method on a square, periodic simulation domain $\mathcal{A}$ of side $L=2 \pi$ with $N_{c}^{2}$ collocation points [18]. In this method, we evaluate the linear terms in Eqs. (2) and (3) in Fourier space and the nonlinear term in real (physical) space, which we then transform to Fourier space. For the Fourier-transform operations we use the FFTW library [19]. A fourth-order, Runge-Kutta scheme, with time step $\Delta t$, is used to evolve these equations in time. Any pseudospectral DNS retains a finite number of Fourier modes; therefore, we introduce the Galerkin projector $\mathcal{P}_{G}$,

$$
\mathcal{P}_{G}[\hat{\psi}(\mathbf{k})]=\theta\left(k_{\max }-|\mathbf{k}|\right) \hat{\psi}(\mathbf{k}),
$$

where $\hat{\psi}$ is the spatial Fourier transform of $\psi, k_{\max }$ is a suitably chosen ultraviolet cutoff, and $\theta(\cdot)$ is the Heaviside function. We use the standard 2/3-dealiasing rule, with $k_{\max }=2 / 3 \times$ $N_{c} / 2$, and we follow Ref. [20] in our treatment of the nonlinear term in the GPE: we first apply $\mathcal{P}_{G}$ on $|\psi|^{2}$ and then again on $\mathcal{P}_{G}\left[|\psi|^{2}\right] \psi$. This ensures global momentum conservation in our DNS, which is essential for the study of collisions between particles [16] and their interactions with the field $\psi$. Thus our Galerkin-truncated GPE (TGPE) becomes

$$
\begin{aligned}
i \hbar \frac{\partial \psi(\mathbf{r}, t)}{\partial t}= & \mathcal{P}_{G}\left[\left(-\frac{\hbar^{2}}{2 m} \nabla^{2}+g \mathcal{P}_{G}\left[|\psi|^{2}\right]\right.\right. \\
& \left.\left.-\mu+\sum_{i=1}^{\mathcal{N}_{0}} V_{\mathcal{P}}\left(\mathbf{r}-\mathbf{q}_{i}\right)\right) \psi(\mathbf{r}, t)\right] .
\end{aligned}
$$

Given our Galerkin-truncation scheme, we can write the force acting on the particle as

$$
\begin{aligned}
\mathbf{f}_{\mathrm{o}, i}= & -\int_{\mathcal{A}}\left[\psi^{*} \mathcal{P}_{G}\left[V_{\mathcal{P}}\left(\mathbf{r}-\mathbf{q}_{i}\right) \nabla \psi\right]\right. \\
& \left.+\psi \mathcal{P}_{G}\left[V_{\mathcal{P}}\left(\mathbf{r}-\mathbf{q}_{i}\right) \nabla \psi^{*}\right]\right] d^{2} x .
\end{aligned}
$$

\section{Units and parameters}

We write the important parameters of the GPE system in the following form: the quantum of circulation $\kappa \equiv h / m \equiv$
$4 \pi \alpha_{0}$, the speed of sound $c=\sqrt{2 \alpha_{0} \tilde{g} \rho_{0}}$, the healing length $\xi=\sqrt{\alpha_{0} /\left(\tilde{g} \rho_{0}\right)}$, where $\tilde{g}=g / \hbar m$, and the mean density $\rho_{0}=$ $\int_{\mathcal{A}} m|\psi|^{2} d \mathbf{r} / \mathcal{A}$; see Appendix A for details. In all our calculations, we set $\rho_{0}=1, c=1$, and $\xi=1.44 d x$, where $d x=$ $L / N_{c}, \quad N_{c}^{2}=128^{2}, \tilde{\mu} \equiv \mu / \hbar=\tilde{g} \rho_{0}, \quad \tilde{V}_{\mathrm{o}} \equiv V_{\mathrm{o}} / \hbar=10 \tilde{g} \rho_{0}$, $d_{\mathrm{p}}=1.5 \xi$, and $\Delta_{\mathrm{E}}=0.062$, except in Sec. III D where we set $c=2$. Energies are in units of $E_{\xi}=2 \alpha \rho_{0}^{2} \tilde{g}=c^{2} \rho_{0}$, which has the dimension [ $M T^{-2}$, as the energies defined in Eqs. (5) and (6) are energies per unit area; to obtain the energy integrated over the whole computation domain $\mathcal{E}_{\mathcal{A}}$, the dimensionless quantity $E$ must be multiplied by $c^{2} \rho_{0} \mathcal{A}$ and the appropriate unit for $\mathcal{E}_{\mathcal{A}}$ is then $c^{2} \xi^{2} \rho_{0}$. The force acting on the particle is expressed in units of $c^{2} \xi \rho_{0}$.

\section{RESULTS}

In order to understand how particles and the field $\psi$ interact in superfluids, we have considered the following illustrative initial configurations in our DNSs.

(1) ICP1: an initial configuration with one particle on which a constant, external force acts for the entire duration of the DNS.

(2) (i) ICP2A: an initial configuration with one particle placed in front of a translating vortex-antivortex pair. (ii) ICP2B: an initial configuration with two particles placed in front of a translating vortex-antivortex pair.

(3) (i) ICP3A: an initial configuration with a single particle moving in the presence of counter-rotating vortex clusters. (ii) ICP3B: an initial configuration with four particles in the presence of counter-rotating vortex clusters.

\section{A. Single-particle dynamics: Constant external force on the particle}

We study the dynamics of a single particle in the superfluid by using the initial configuration ICP1, where an external force acts on the particle; in particular, we examine the dependence of this dynamics on $\mathcal{M}$. We achieve this by first preparing an initial state with a single particle at rest by using the advectiveGinzburg-Landau equation (ARGLE) (see Appendix B). We use this initial state in the GPE and switch on the external force $\mathbf{F}_{\text {ext }}=F_{0} \hat{\mathbf{x}}$, where $F_{0}$ is constant in time. We now describe the dynamics of the heavy $(\mathcal{M}>1)$, neutral $(\mathcal{M}=1)$, and light particles $(\mathcal{M}<1)$ for the initial configuration ICP1.

Heavy particle. We apply an external force $\mathbf{F}_{\mathrm{ext}}=$ $0.28 c^{2} \xi \rho_{0} \hat{\mathbf{x}}$ on the heavy particle. In Fig. 1 we show pseudocolor plots of $\rho(\mathbf{r})$, at different instants of time, to illustrate its spatiotemporal evolution; the particle and vortices appear as blue disks in which $\rho=0$, with large and small diameters, respectively. In Fig. 2(a) we show the temporal evolution of the $x$ and the $y$ components of the particle velocity $u_{\mathrm{o}, \mathrm{x}}$ (blue solid curve) and $u_{\mathrm{o}, \mathrm{y}}$ (red dashed curve), respectively.

The particle starts to move from rest through the superfluid, without disturbing the latter, until the critical velocity $u_{c} / c \simeq$ 0.47 is reached at $c t / \xi=3.313 \times 10^{4}$. When the particle velocity $\simeq u_{c}$, a vortex-antivortex pair emerges, with a positive vortex at the top and a negative vortex at the bottom of the particle, but both still attached to, and comoving with, the particle; the particle slows down slightly [Fig. 1(b)]. Subsequently, the vortex-antivortex pair gets detached from 

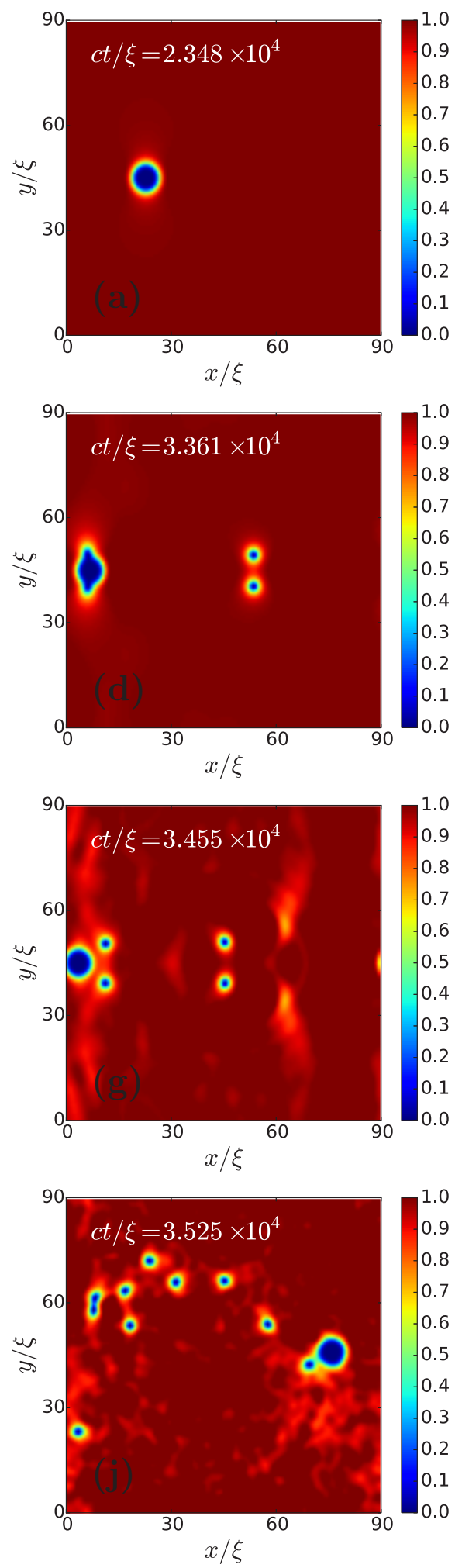
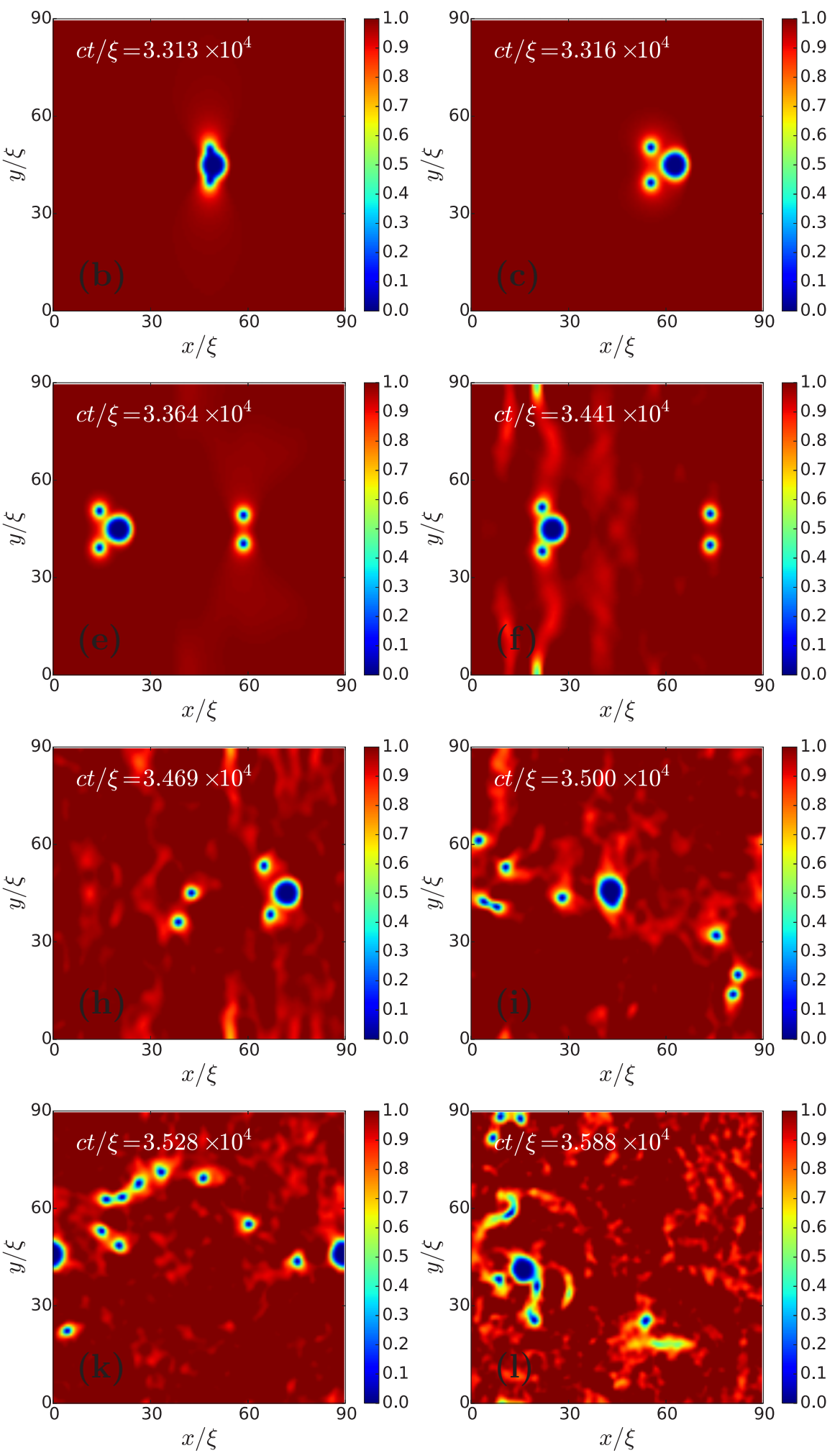

FIG. 1. Spatiotemporal evolution of the density field $\rho(\mathbf{r}, t)$ shown via pseudocolor plots, illustrating the dynamics of a heavy particle, when a constant external force $\mathbf{F}_{\text {ext }}=0.28 c^{2} \xi \rho_{0} \hat{\mathbf{x}}$ acts on it (initial configuration ICP1). The particle appears as a large blue patch and the vortices as blue dots (for details see text, Sec. III A).

the particle; it is oriented perpendicular to, and moves along, the $x$ direction at a much reduced velocity compared to that of the particle [Fig. 1(c)]. Given its large velocity, the particle moves ahead of the slowly moving vortex-antivortex pair and, because of the periodic boundary conditions we use, it comes back and approaches the vortex-antivortex pair from behind. The particle passes through the vortex-antivortex pair, during which passage the positive and the negative vortices 

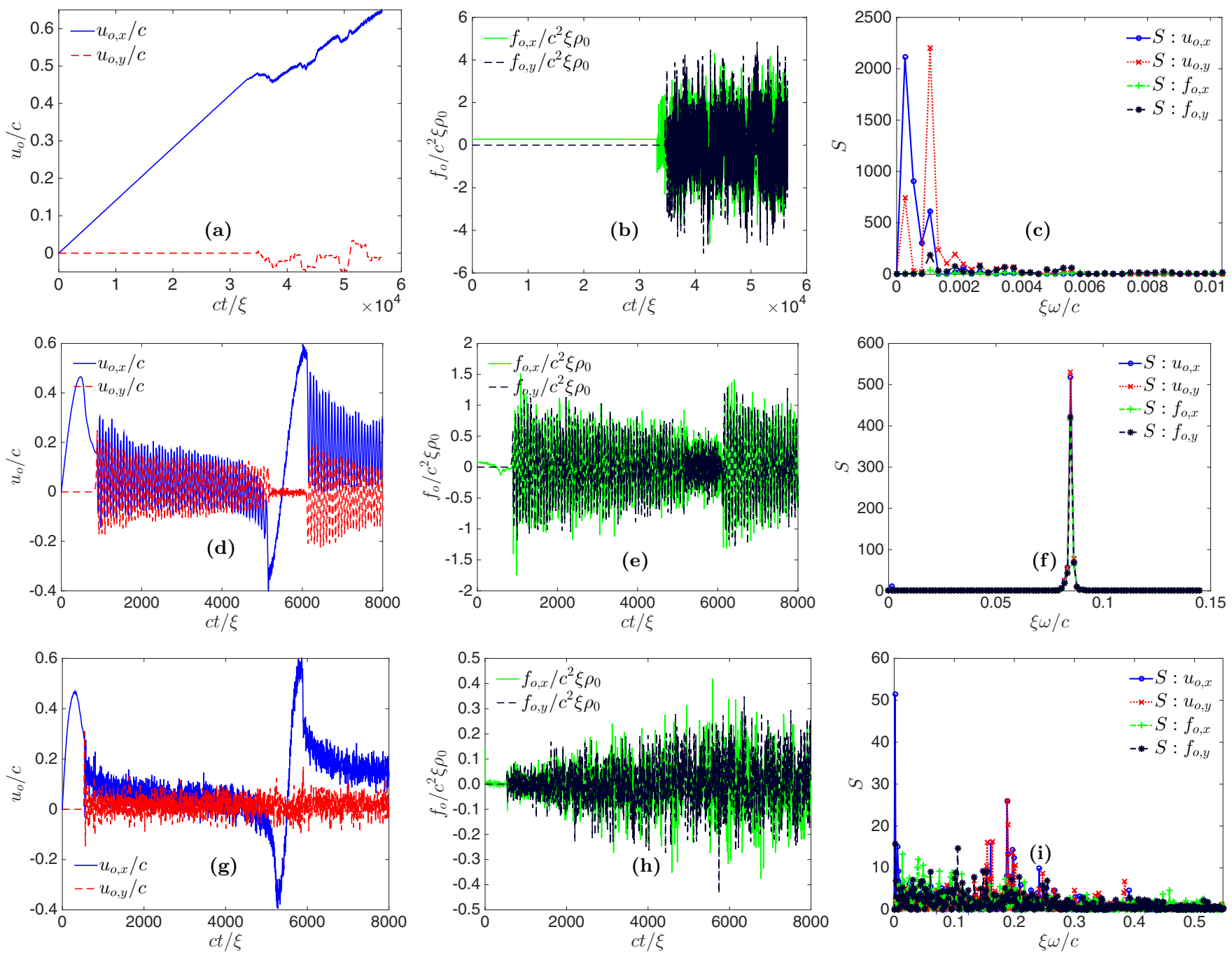

FIG. 2. Plots of the Cartesian components of (a) velocity $u_{\mathrm{o}, \mathrm{x}}$ and $u_{\mathrm{o}, \mathrm{y}}$, (b) force $f_{\mathrm{o}, \mathrm{x}}$ and $f_{\mathrm{o}, \mathrm{y}}$, and (c) power spectra of the quantities time series in (a) and (b), for the heavy particle $\left(\mathcal{M}=374, \mathbf{F}_{\text {ext }}=0.28 c^{2} \xi \rho_{0} \hat{\mathbf{x}}\right)$. Plots in (d)-(f) and (g)-(i) are the analogs of plots in (a)-(c), for the neutral $\left(\mathcal{M}=1, \mathbf{F}_{\text {ext }}=0.14 c^{2} \xi \rho_{0} \hat{\mathbf{x}}\right)$ and $\operatorname{light}\left(\mathcal{M}=0.0374, \mathbf{F}_{\text {ext }}=0.14 c^{2} \xi \rho_{0} \hat{\mathbf{x}}\right)$ particles, respectively. Power spectra, denoted generically by $S(\omega)$, of the time series of $u_{\mathrm{o}, \mathrm{x}}, u_{\mathrm{o}, \mathrm{y}}, f_{\mathrm{o}, \mathrm{x}}$, and $f_{\mathrm{o}, \mathrm{y}}$ are plotted versus the angular frequency $\omega$ for the above three cases.

glide, respectively, along the upper and lower half of the circumference of the particle; such an interaction is also associated with the initial increase (which is followed by a decrease) in the particle velocity because of the reinforcing nature of the velocity field in the region in between the vortex and the antivortex that constitute the pair. Moreover, this interaction of the particle and the vortex-antivortex pair leads to the generation of sound waves. The particle subsequently sheds another vortex-antivortex pair [Figs. 1(d) and 1(e)] and then it interacts with two vortex-antivortex pairs; this is accompanied by an even greater emission of sound waves than in the case of one vortex-antivortex pair. Afterwards, the presence of sound waves during the interaction of the particles with the vortex-antivortex pairs results in deflections of the latter from their trajectories [Fig. 1(h)]; at the same time, small fluctuations are induced in the particle velocity [see Fig. 2(a)].

The subsequent motions of the particle and the vortexantivortex pairs become complicated [Figs. 1(i)-1(1)]. Vortexantivortex pairs are emitted whenever the particle velocity exceeds the critical velocity $u_{c}$. Many more such pairs are shed by the particle and, at several instances of the shedding of a vortex-antivortex pair, one of the vortices is trapped on the particle for a short duration of time. Moreover, the vortices and antivortices frequently annihilate and produce sound waves during this annihilation.

The observation that vortex-anitvortex pairs are emitted at $u_{\mathrm{o}}>u_{c}$ does not mean that critical velocity has been modified. This just signifies that the force acting on the particle is large enough to accelerate it beyond the critical velocity $u_{c}$. The particle does not emit vortices below the critical velocity, but it can (and does) emit vortices above the critical velocity.

Figure 2(b) shows that the force exerted by the superfluid on the particle [see Eqs. (4) and (17)] exhibits large fluctuations after the critical velocity is reached. In Fig. 2(c) we plot the power spectra of the time series of the Cartesian components $f_{\mathrm{o}, \mathrm{x}}$ (green solid curve) and $f_{\mathrm{o}, \mathrm{y}}$ (black dashed curve); these show that many frequencies appear in these spectra. The video M1 in the Supplemental Material [21] illustrates the 

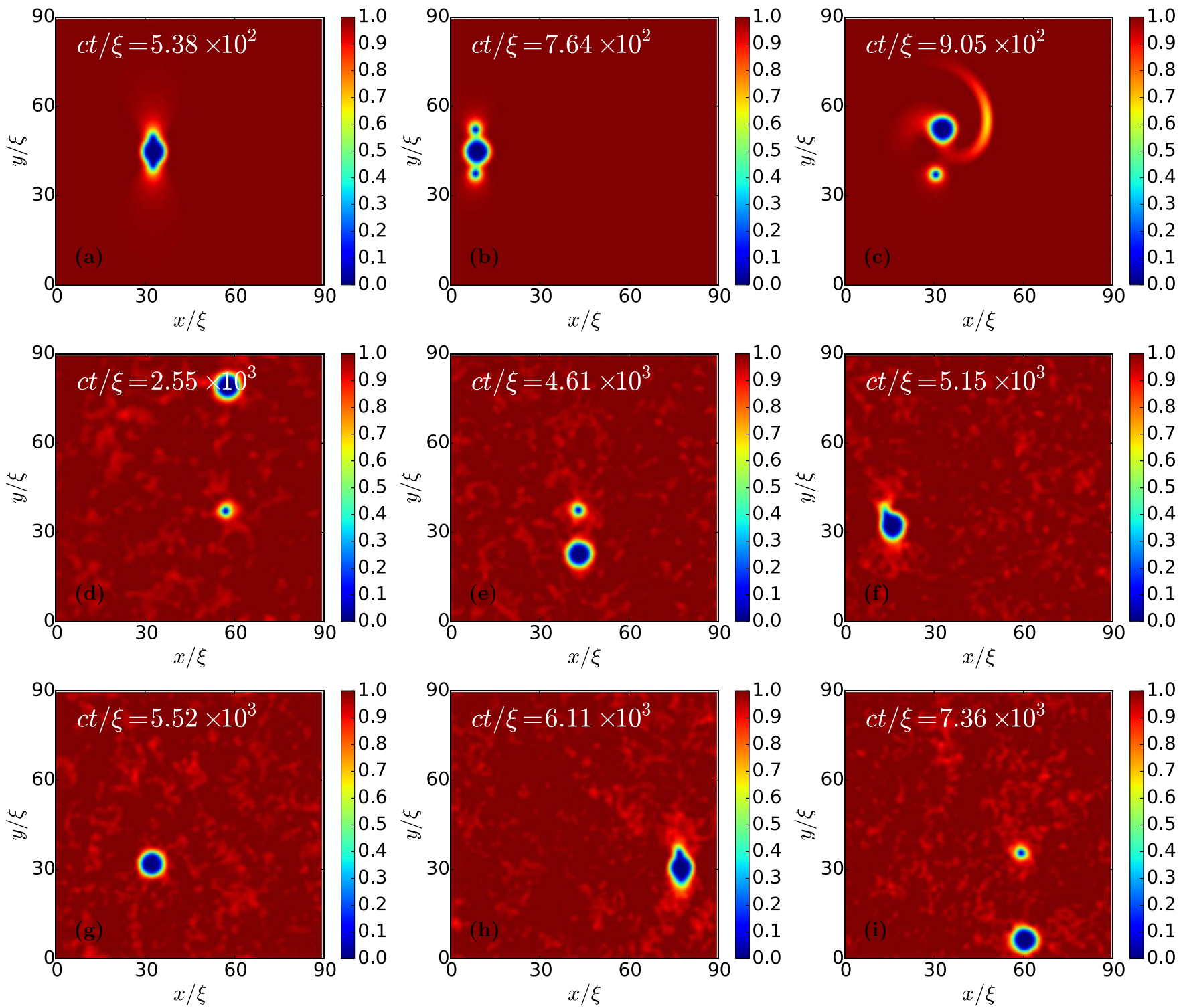

FIG. 3. Spatiotemporal evolution of the density field $\rho(\mathbf{r}, t)$ shown via pseudocolor plots, illustrating the dynamics of a neutral particle, when a constant external force $\mathbf{F}_{\text {ext }}=0.14 c^{2} \xi \rho_{0} \hat{\mathbf{x}}$ acts on it (initial configuration ICP1). The particle appears as a large blue patch and the vortices as blue dots (for details see text, Sec. III A).

spatiotemporal evolution of a forced heavy particle in a superfluid. This video, the time series of $u_{\mathrm{o}, \mathrm{x}}, u_{\mathrm{o}, \mathrm{y}}, f_{\mathrm{o}, \mathrm{x}}$, and $f_{\mathrm{o}, \mathrm{y}}$, and their power spectra [Figs. 2(c), 2(f), and 2(i)] show that, after the first vortex-antivortex pair has been emitted, the motion of the particle can be temporally chaotic, for the heavy particle.

Neutral particle. To study the dynamics of a neutral particle in the superfluid, we use the initial configuration ICP1. We apply an external force $\mathbf{F}_{\text {ext }}=0.14 c^{2} \xi \rho_{0} \hat{\mathbf{x}}$ on the particle. In Figs. 3(a)-3(i) we show, via pseudocolor plots, the spatiotemporal evolution of $\rho(\mathbf{r}, t)$; the particle appears as a large blue patch on these plots. The particle accelerates under the influence of the external force and its velocity reaches a maximum at $c t / \xi \simeq 467$, before starting to decrease [see Fig. 2(d)]; this maximum of velocity is also the critical velocity $u_{c} \simeq$ $0.47 c$, where a vortex-antivortex pair is formed. Figure 3(a) shows the vortex-antivortex pair still attached to the particle at $c t / \xi=5.38 \times 10^{2}$, as an extension along the $y$ direction of the particle (blue patch). Subsequently, the vortex-antivortex pair is detached from the particle [Fig. 3(b)], but the particle and the vortex-antivortex-pair assembly (henceforth PVA complex) becomes unstable, with respect to motion transverse to the direction of $\mathbf{F}_{\text {ext }}$ at $c t / \xi \simeq 7.64 \times 10^{2}$, and oscillatory modes are excited, as we show in Fig. 2(d), where the Cartesian components $u_{\mathrm{o}, \mathrm{x}}$ and $u_{\mathrm{o}, \mathrm{y}}$ of the particle velocity exhibit modulated oscillations. In Fig. 2(f), the power spectra of both $u_{0, \mathrm{x}}$ and $u_{o, y}$ show one large peak and two or three small peaks; the former is associated with the main temporal oscillation and the latter with the modulation. Figure 2(e) shows that similar oscillations are present in the Cartesian components of the force exerted by the superfluid on the particle. As a result of this instability, at $c t / \xi \simeq 9.05 \times 10^{2}$ the particle is trapped on the 
positive (upper) vortex [Fig. 3(c)]; this is accompanied by an intense emission of sound waves. The particle, trapped on the positive vortex, and the negative (lower) vortex move together, with both aligned roughly perpendicular to the direction of motion [Fig. 3(d)]; the separation between the two increases, principally because the positive vortex moves away and takes the trapped particle along with it. Because of our periodic boundary conditions, the positive vortex (and the particle trapped by it) comes back from below (the $y$ direction). The direction of the velocity field, in the small region in between the particle and the vortex, generated by the vortex-antivortex pair, is reversed (from $+\hat{\mathbf{x}}$ to $-\hat{\mathbf{x}}$ ). When the negative vortex and the positive vortex (and the trapped particle) are close enough to each other, then the field generated by the pair is so strong that the PVA complex reverses its direction of motion at $c t / \xi \simeq$ $4.61 \times 10^{3}$ [Fig. 3(e)]. When the two vortices are very close together [see Fig. 3(f)], they annihilate at $c t / \xi=5.15 \times 10^{3}$, while the particle moves predominantly in the $-\hat{\mathbf{x}}$ direction. Soon thereafter, the acceleration, because of the external force, reverses the direction of motion once again and the particle begins to move predominantly in the $\hat{\mathbf{x}}$ direction as we show in Fig. $3(\mathrm{~g})$ at $c t / \xi=5.52 \times 10^{3}$. The particle velocity increases, reaches a maximum value $u_{\mathrm{o}, \mathrm{x}}=0.57 c>u_{c}$, and again a vortex-antivortex pair is formed at $t=6.11 \times 10^{3}$; initially, this pair is attached to the particle. At $c t / \xi \simeq 6.14 \times 10^{3}$ the particle gets trapped on the negative (lower) vortex and the cycle of particle and vortex motions, described above, is repeated again [see Fig. 2(d) for $t \gtrsim 6.14 \times 10^{3}$ and Fig. 3(i)]. Video M2 [21] gives the complete spatiotemporal evolution of the particle and $\rho(\mathbf{r}, t)$.

Light particle. We now describe the dynamics of a light particle in the superfluid by using the initial configuration ICP1; the particle is accelerated by applying an external force $\mathbf{F}_{\text {ext }}=0.14 c^{2} \xi \rho_{0} \hat{\mathbf{x}}$ on it. Figures S1(a)-(i) and Video M3 in the Supplemental Material [21] summarize the spatiotemporal evolution of the field $\rho(\mathbf{r}, t)$ for the light-particle case. A comparison of the $x$ component of the particle velocity $u_{\mathrm{o}, \mathrm{x}}$ in Figs. 2(d) and 2(g), and a comparison of the field $\rho(\mathbf{r}, t)$ in Figs. 3 and Fig. S1 in the Supplemental Material [21], shows that the dynamics of the light particle is similar to, but not exactly the same as, that of the neutral particle. A major feature which distinguishes their dynamics is the presence of a broad range of frequencies in the power spectra of $\mathbf{u}_{\mathrm{o}}$ and $\mathbf{f}_{\mathrm{o}}$ of the former case; this indicates that the motion of the light particle is chaotic, in contrast to that of the neutral particle, whose dynamical evolution is periodic in time.

The chaotic nature of the particle dynamics is enhanced when we increase the amplitude of the external force acting on the particle, as we show in Fig. 18 in the Appendixes; in particular, now the power spectra of the Cartesian components of $\mathbf{u}_{\mathrm{o}}$ and $\mathbf{f}_{\mathrm{o}}$ have a broad range of frequencies for all the three types of particles. We can understand the motion of the particles by using the concept of the hydrodynamical mass (or effective mass) $m_{*}=\partial P_{\text {ext }} / \partial u_{\mathrm{o}}\left(P_{\text {ext }}=F_{\text {ext }} t\right)$. To begin with, there is no drag force on the particle; but the particle still transfers momentum to the fluid by virtue of the increase in its effective mass; this becomes very large (ideally infinity) at $u_{\mathrm{o}}=u_{c}$, where a vortex-antivortex pair is formed. Note that, when a vortex-antivortex pair is formed, the plot of $u_{\mathrm{o}, \mathrm{x}}$ versus time has a maximum [see Fig. 2(d)], i.e., the acceleration in the

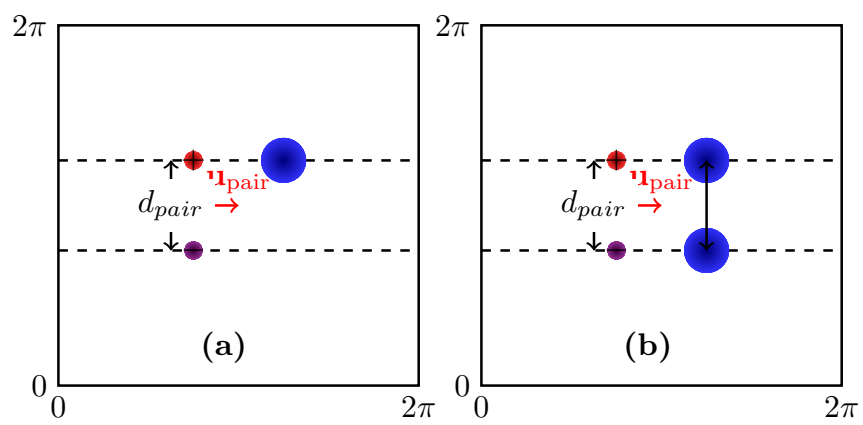

FIG. 4. Schematic diagrams (a) and (b) illustrate the initial configurations ICP2A (single particle case) and ICP2B (two particles case), respectively, which we use to study the interaction of the particles (blue disks) with the superfluid field, by placing them in the path of a translating vortex-antivortex pair (represented by small red and purple disks).

$x$ direction vanishes, even though the force $F_{\text {ext }}$ is nonzero. Therefore, the effective mass $m_{*}$ diverges when the vortexantivortex pair is created. After this, $m_{*}$ becomes negative and the particle slows down; this is more apparent in the cases of the neutral and the light particles than for a heavy particle.

\section{B. Interaction of particles with a translating vortex-antivortex pair}

Single particle. Next we study the interaction of a single particle with a vortex-antivortex pair for heavy, neutral, and light particles by using the initial configuration ICP2A, in which we place a particle in the path of the positive vortex of the translating vortex-antivortex pair. In order to implement this, we prepare a state with a stationary particle at $(1.5 \pi / \xi, 1.257 \pi / \xi)$ and then combine it with a state corresponding to a vortexantivortex pair of size $d_{\text {pair }} \simeq 23 \xi$ and which translates with a velocity $\mathbf{u}_{\text {pair }}=0.074 c \hat{\mathbf{x}}$; see the schematic diagram in Fig. 4(a) and Appendix D 1 for preparation details.

When the vortex-antivortex pair approaches the heavy particle, the positive (upper) vortex glides over the particle, which leads to an exchange of momentum, so the vortex-antivortex pair is deflected from its path, and it acquires a small velocity in the $-\hat{\mathbf{y}}$ direction. This interaction leads to the production of sound waves. Subsequently, when the negative vortex (of the vortex-antivortex pair) comes near the heavy particle, in the presence of sound waves, it is finally trapped on the particle. During the trapping of the particle on the negative vortex, a large amount of acoustic energy is released into the system. This sequence of events is illustrated by the pseudocolor plots of Figs. 5(a)-5(l) and Video M4 [21]. Figures 6(a) and 6(b) show plots of $u_{0, x}$ and $u_{0, y}$ versus time, respectively, for heavy, neutral, and light particles. The trapped heavy particle executes oscillatory motion while drifting [see Figs. 6(a) and 6(b) for $c t / \xi \gtrsim 3000$ ] and the positive vortex now revolves around the particle that is trapped on the negative vortex.

When a translating vortex-antivortex pair approaches neutral or light particles, they feel the flow around the positive (upper) vortex more strongly than did the heavy particle. The neutral and light particles are pushed out and they move around the positive vortex before getting trapped on the positive 

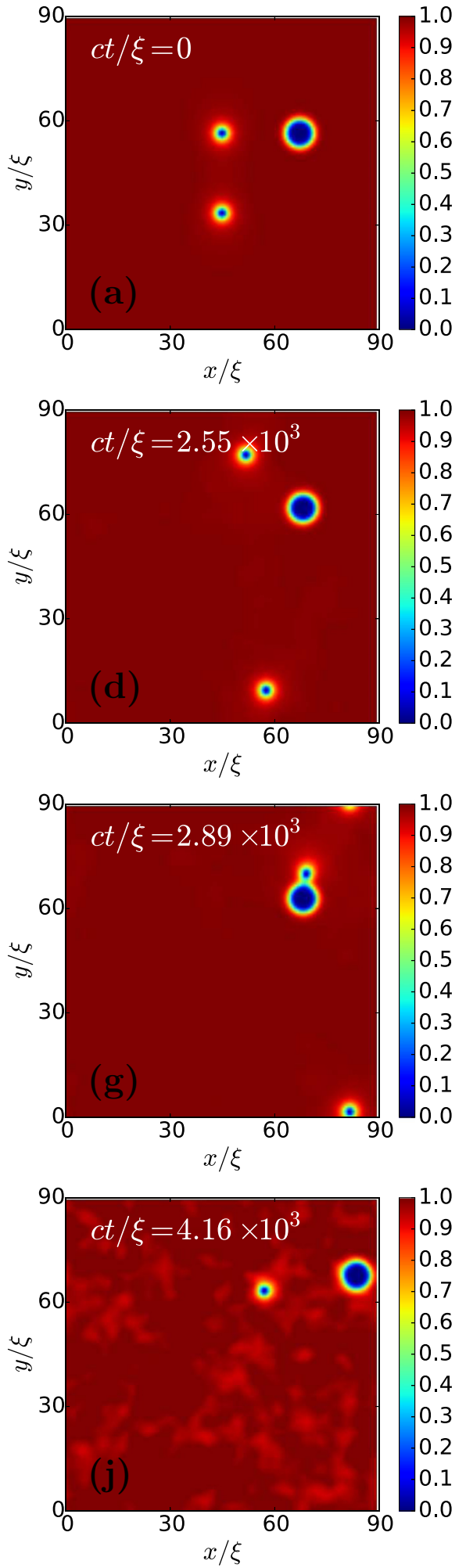
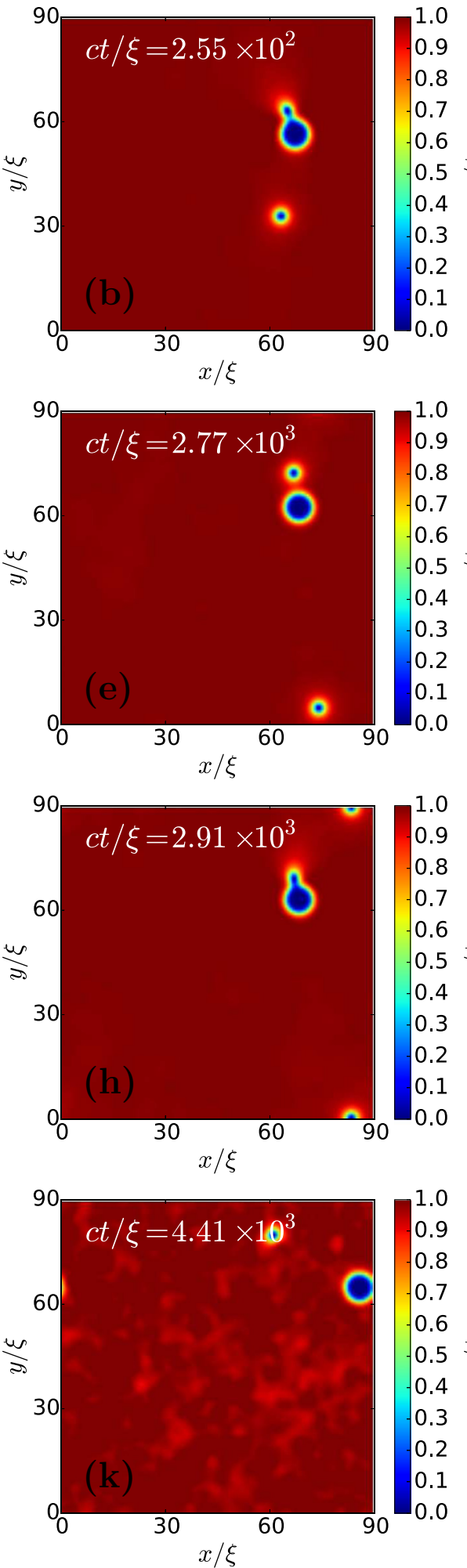
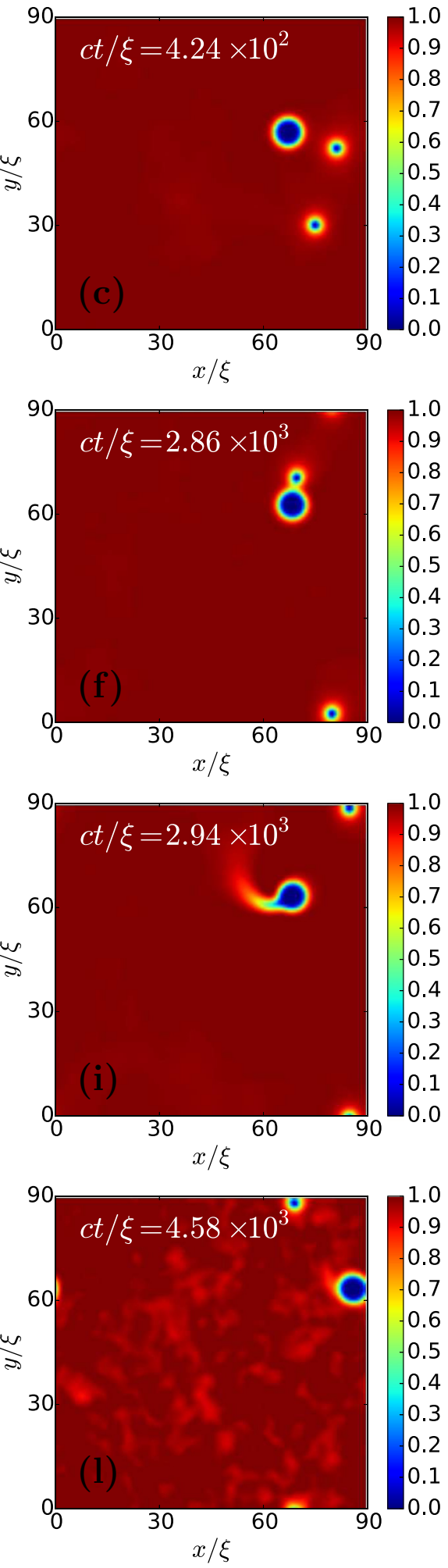

FIG. 5. Spatiotemporal evolution of the density field $\rho(\mathbf{r}, t)$ shown via pseudocolor plots, for a heavy particle placed in the path of the positive (upper) vortex of a translating vortex-antivortex pair (initial configuration ICP2A).

vortex; in Fig. 6(c), inset of (c) and (d), we show this initial motion by plotting the particle velocity components $u_{\mathrm{o}, \mathrm{x}}, u_{\mathrm{o}, \mathrm{y}}$ and the trajectories, respectively. The response of the light particle is most dramatic: while moving around the positive vortex, it is pushed almost to the back of the vortex $(0 \lesssim c t / \xi \lesssim$ $3.11 \times 10^{2}$ ) before getting trapped on it; see Figs. 19(a)-19(d). The pseudocolor plots of Figs. 7(a)-7(f) and 19(a)-19(i) in the Appendixes show the density field at different instants 

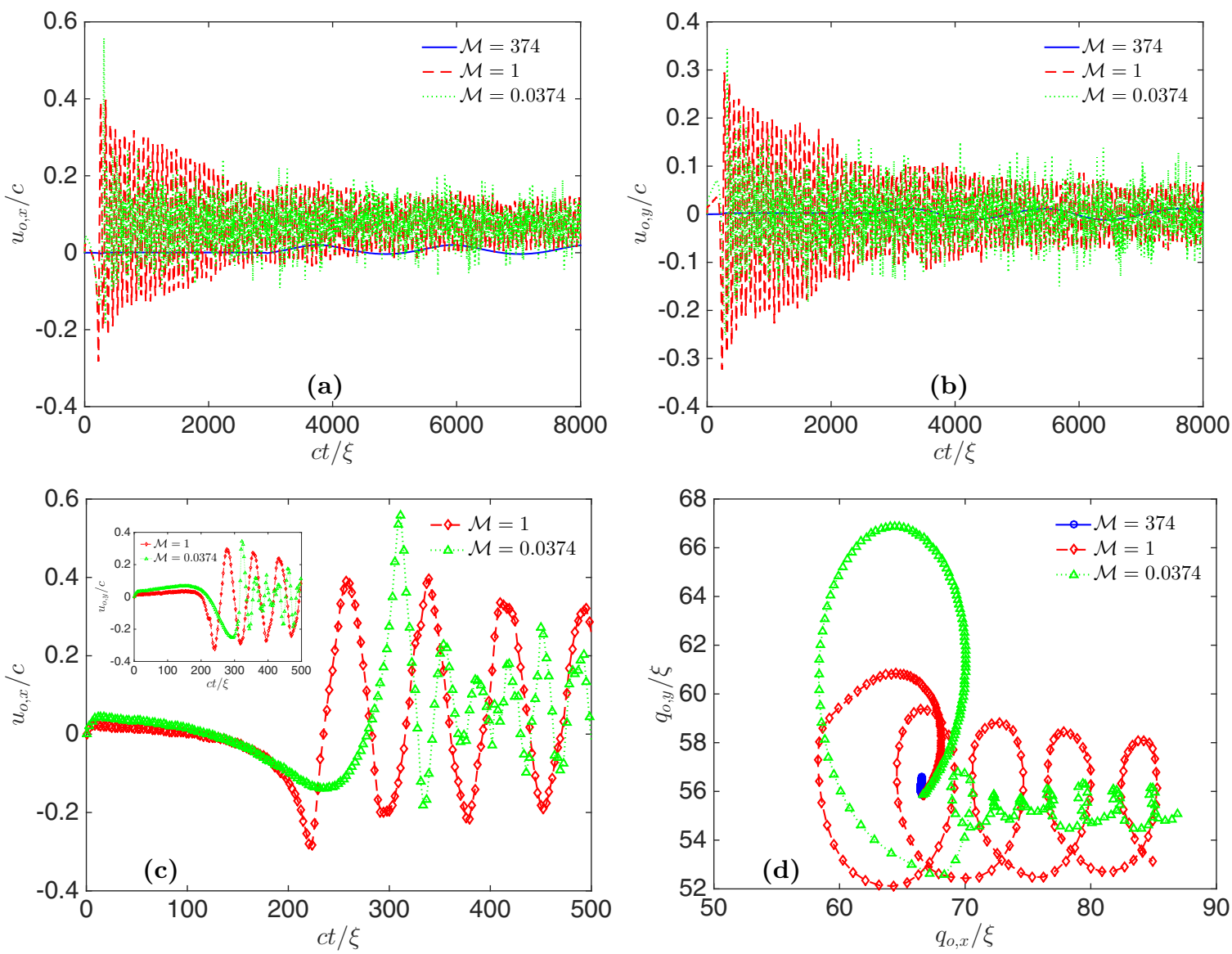

FIG. 6. Plots vs time $t$ of (a) $u_{\mathrm{o}, \mathrm{x}}$ and (b) $u_{\mathrm{o}, \mathrm{y}}$ for heavy $(\mathcal{M}=374$, blue curve), neutral $(\mathcal{M}=1$, red curve), and light $(\mathcal{M}=0.0374$, green curve) particles, placed in the path of the positive (upper) vortex of a translating vortex-antivortex pair (initial configuration ICP2A). The initial stage of motion is emphasized by separately plotting $u_{\mathrm{o}, \mathrm{x}}$ in (c) and $u_{\mathrm{o}, \mathrm{y}}$ in the inset for the neutral and light particles; (d) contains the trajectories $\left(q_{\mathrm{o}, \mathrm{x}} / \xi, q_{\mathrm{o}, \mathrm{y}} / \xi\right)$ for the three particle types during this time.

of time for neutral and light particles, respectively; for the complete spatiotemporal evolution, see Videos M5 and M6 in the Supplemental Material [21]. When the neutral and the light particles get trapped on the positive vortex, there is a sudden change in their velocities, as we show in Figs. 6(a)-6(c) at $c t / \xi \simeq 2.00 \times 10^{2}$; this results in large fluctuations. Neutral particles exhibit modulated oscillations, whereas light particles display chaotic temporal evolution. In Figs. 8(a)-8(c) we plot the energy time series for the heavy, neutral, and light particles, respectively. These plots illustrate the continual exchange of energy between the particle and the superfluid.

Two particles. We now investigate the interaction of two particles with a vortex-antivortex pair. For this we use the initial configuration ICP2B, in which we place the two particles Pt : 1 and Pt : 2 at a distance, in front of the positive and the negative vortices of a translating vortex-antivortex pair [see the schematic diagram in Fig. 4(b)]. As in the case of a single particle, we use the ARGLE to prepare a state with two stationary particles Pt : 1 and $\mathrm{Pt}: 2$ at $(1.5 \pi / \xi, 1.257 \pi / \xi)$ and $(1.5 \pi / \xi, 0.743 \pi / \xi)$, respectively; this state is then combined with a state corresponding to a vortex-antivortex pair of size $d_{\text {pair }} \simeq 23 \xi$ which translates with a velocity $\mathbf{u}_{\text {pair }}=0.074 c \hat{\mathbf{x}}$ (see Appendix D 1 for preparation details). We use the above initial configuration to study the interaction of the vortexantivortex pair with heavy, neutral, and light particles.

In Figs. 9(a)-9(e) we show that, when the vortex-antivortex pair approaches the two symmetrically placed heavy particles, the positive (upper) vortex and the negative (lower) vortex glide along the circumferences of $\mathrm{Pt}: 1$ and $\mathrm{Pt}: 2$, respectively; thereafter, the vortex-antivortex pair continues to translate in the $\hat{\mathbf{x}}$ direction. The interaction of the vortex-antivortex pair with these particles leads to the transfer of momentum to the latter and these particles start moving slowly [see Figs. $10(\mathrm{a})-10(\mathrm{~d})$ for $c t / \xi \simeq 2.26 \times 10^{2}$ ]. Because of our periodic boundary conditions, the translating vortex-antivortex pair comes back and again glides along the particle boundaries, which are still in the path of translation of this pair [see Figs. 9(f)-9(i)]. The particles move away from the vortices, as the vortex-antivortex pair moves beyond them [see Figs. 10(a)10 (d) for $c t / \xi \gtrsim 1.41 \times 10^{3}$ ]. At later times, the separation between the particles is wide enough for the vortex-antivortex pair to pass through the region in between the particles without any significant obstruction [see Figs. 9(j)-9(1)]. However, the plots of the particle-velocity components versus times show jumps when the vortex-antivortex pair passes through the region in between the particles. The Video M7 [21] illustrates 

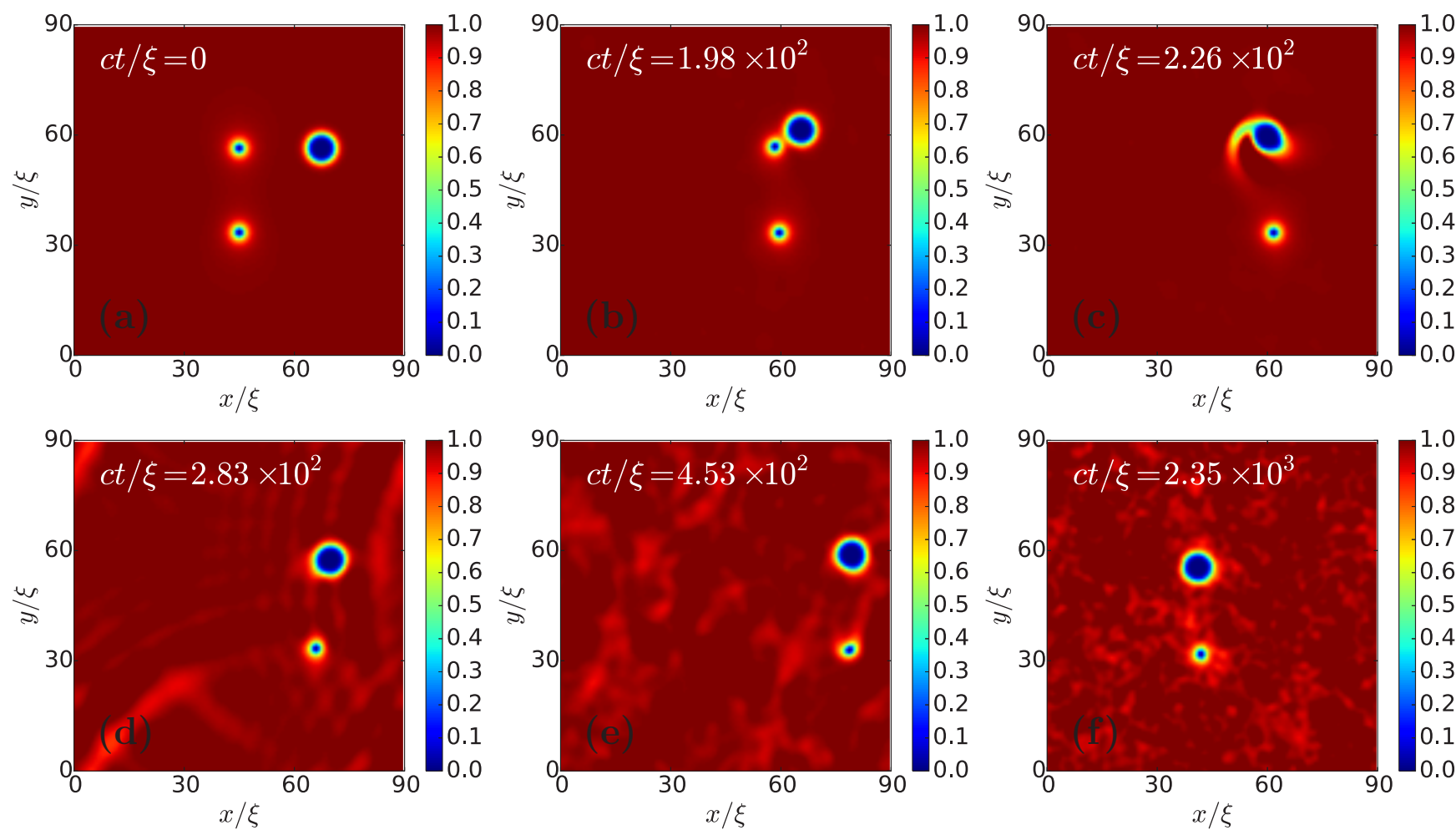

FIG. 7. Spatiotemporal evolution of the density field $\rho(\mathbf{r}, t)$ shown via pseudocolor plots, for a neutral particle placed in the path of the positive (upper) vortex of a translating vortex-antivortex pair (initial configuration ICP2A).

the complete spatiotemporal evolution of the particles and the density field $\rho(\mathbf{r}, t)$.

When the translating vortex-antivortex pair approaches symmetrically placed neutral or light particles, the particles $\mathrm{Pt}: 1$ and $\mathrm{Pt}: 2$ get trapped on the positive and the negative vortices, respectively. The trapping of the two neutral (light) particles here is similar to the trapping of a single neutral (light) particle placed in front of a translating vortex-antivortex pair. After the particles are trapped on the vortices, the twoparticle-vortex-antivortex-pair complex continues to translate in the $\hat{\mathbf{x}}$ direction, but the particles now exhibit fluctuations. The pseudocolor plots of Figs. S2(a)-(f) and S3(a)-(f) and the Videos M8 and M9 in the Supplemental Material [21] summarize the spatiotemporal evolution of the density field $\rho(\mathbf{r}, t)$ for the neutral and the light particles, respectively. The fluctuations in the case of the neutral particle are temporally periodic, with some modulation, whereas those in the case of the light particle are chaotic [see Figs. S4(a)-(d) (neutral particle) and S5(a)-(d) (light particle) in the Supplemental Material [21] for details].

\section{Single-particle dynamics in the presence of counter-rotating vortex clusters}

To study the dynamics of a single neutral particle in the presence of counter-rotating vortex clusters we generate the initial configuration ICP3A in three steps: (1) we use the ARGLE to prepare a state with a particle at $(\pi / \xi, \pi / \xi)$ moving with velocity $\mathbf{u}_{o}=0.1(1 / \sqrt{2},-1 / \sqrt{2}) c$ (see Appendix D2 for
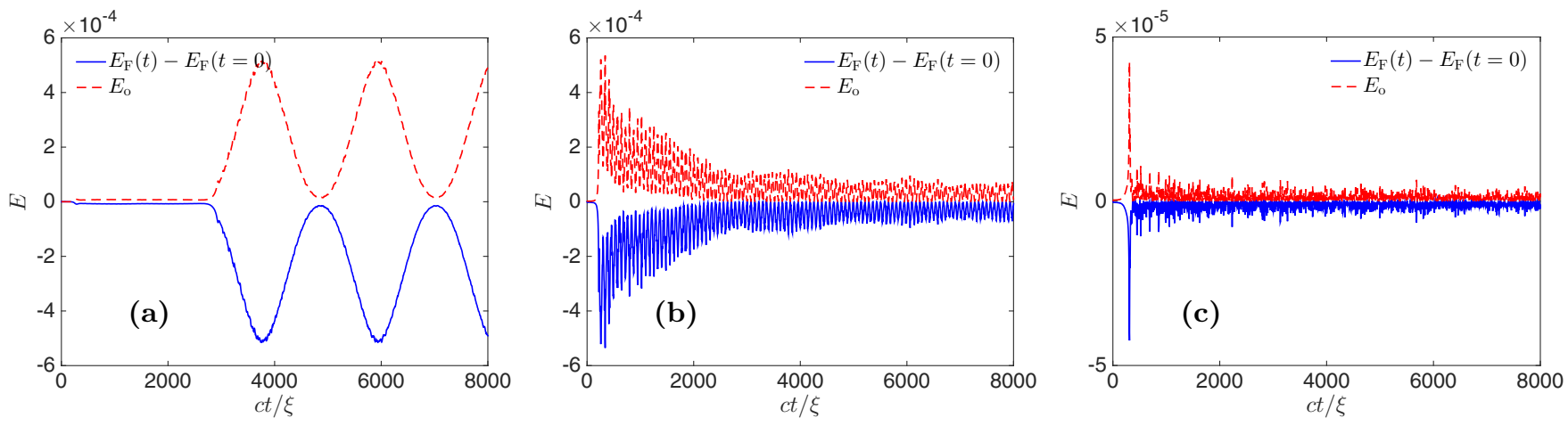

FIG. 8. Plots vs time $t$ of the energy components $\delta E_{\text {field }}=E_{\mathrm{F}}(t)-E_{\mathrm{F}}(t=0)$ (blue solid curve) and $E_{\mathrm{o}}$ (red dashed curve) for (a) heavy, (b) neutral, and (c) light particles, placed in the path of the positive (upper) vortex of a translating vortex-antivortex pair (initial configuration ICP2A). 

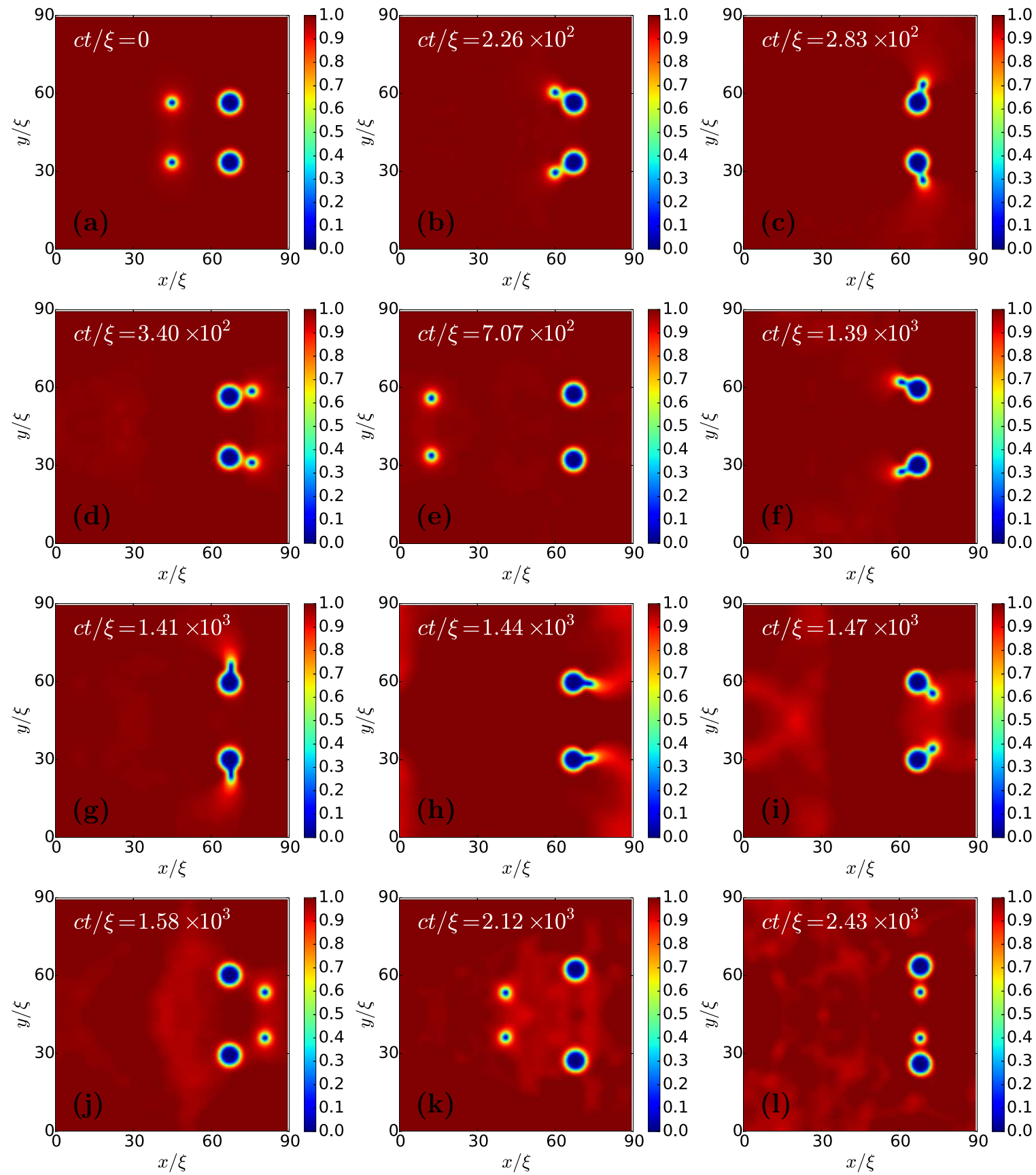

FIG. 9. Spatiotemporal evolution of the density field $\rho(\mathbf{r}, t)$ shown via pseudocolor plots, for two heavy particles placed in the path of the positive (upper) and negative (lower) vortices, respectively, of a translating vortex-antivortex pair (initial configuration ICP2B).

details); (2) we then use the ARGLE to prepare two positive and two negative vortex clusters, where each cluster has 12 vortices of the same sign, and the positive and the negative clusters rotate in opposite directions (for preparation details see the Appendix); (3) the states obtained in the steps (1) and (2) are combined together, by multiplying their wave functions. The initial configuration so prepared is then used in the GPE. By design, we prepare the state with counter-rotating vortex clusters in a state that is not the ground state; therefore, under the TGPE dynamics, the clusters expand and interact with neighboring clusters; this results in a flow with a complex distribution of vortices. Thus this initial state allows us to study a neutral particle in a state that displays superfluid turbulence.
In Figs. 11(a)-11(f) we show the spatiotemporal evolution of the filtered vorticity field; the particle is represented by a black disk here. Figures 11(a)-11(c) show that the vortex clusters expand quickly and interact with their neighboring clusters. At $c t / \xi \simeq 4.53 \times 10^{1}$ the particle sheds a vortexantivortex pair, while moving towards the negative vortex cluster in the right bottom corner of the simulation domain; the particle gets trapped on a nearby negative vortex and it is dragged inside the cluster; at this time, its velocity shoots up to $u_{\mathrm{o}} \sim 0.8 c$. The vortex density decreases as the system evolves because of the annihilation of the vortices and the anitvortices [see Figs. 11(d) and 11(e)]. The Video M10 [21] illustrates the dynamics of a neutral particle in 

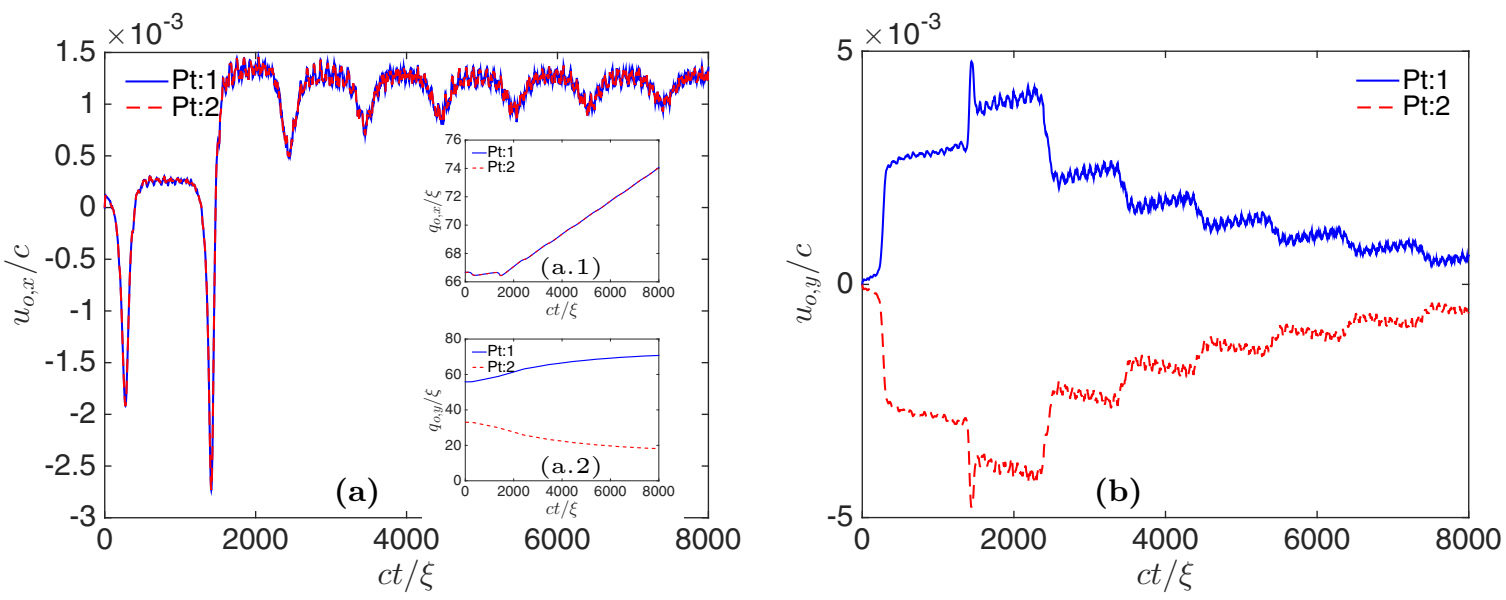

FIG. 10. Plots of (a) $u_{\mathrm{o}, \mathrm{x}}$ and (b) $u_{\mathrm{o}, \mathrm{y}}$ versus time for two heavy particles Pt : 1 (blue solid curve) and Pt : 2 (red dashed curve), placed in the path of the positive (upper) and negative (lower) vortices, respectively, of a translating vortex-antivortex pair (initial configuration ICP2B). Insets: plots of (a.1) $q_{\mathrm{o}, \mathrm{x}}$ and (a.2) $q_{\mathrm{o}, \mathrm{y}}$ vs time. The values of $q_{\mathrm{o}, \mathrm{x}}$ and $q_{\mathrm{o}, \mathrm{y}}$ are not $\bmod 2 \pi$; i.e., if the particle goes around our periodic simulation domain once, say in the $\hat{\mathbf{x}}$ direction, then the value of $q_{\mathrm{o}, \mathrm{x}}$ is its value in the box plus $2 \pi$.

the presence of counter-rotating vortex clusters. Figure 12 shows that the trajectory of the particle (denoted by a series of blue triangles), in the presence of vortices, is complex. The spacing between successive triangles is large (small) when the particle velocity is large (small). During the motion the particle switches from one vortex to another and its direction of motion keeps changing because of its interactions with neighboring vortices. The regions with a high density of circles on the trajectory plot in Fig. 12 occur when the area around the particle is free of vortices or at late times when the overall vortex density has decreased considerably. Figure 13(a) shows that $u_{0, \mathrm{x}}$ and $u_{\mathrm{o}, \mathrm{y}}$ exhibit chaotic fluctuations; in Fig. 13(b) we show plots of the energies that illustrate the exchange of energy between the particle and the superfluid. Energy spectra of the incompressible kinetic energy, plot not included here, shows the spread of kinetic energy over the full range of scales, similar to the case of a turbulent flow [18].

\section{Many-particle dynamics in the presence of counter-rotating vortex clusters}

We study the dynamics of four neutral particles in the presence of small, counter-rotating vortex clusters as an
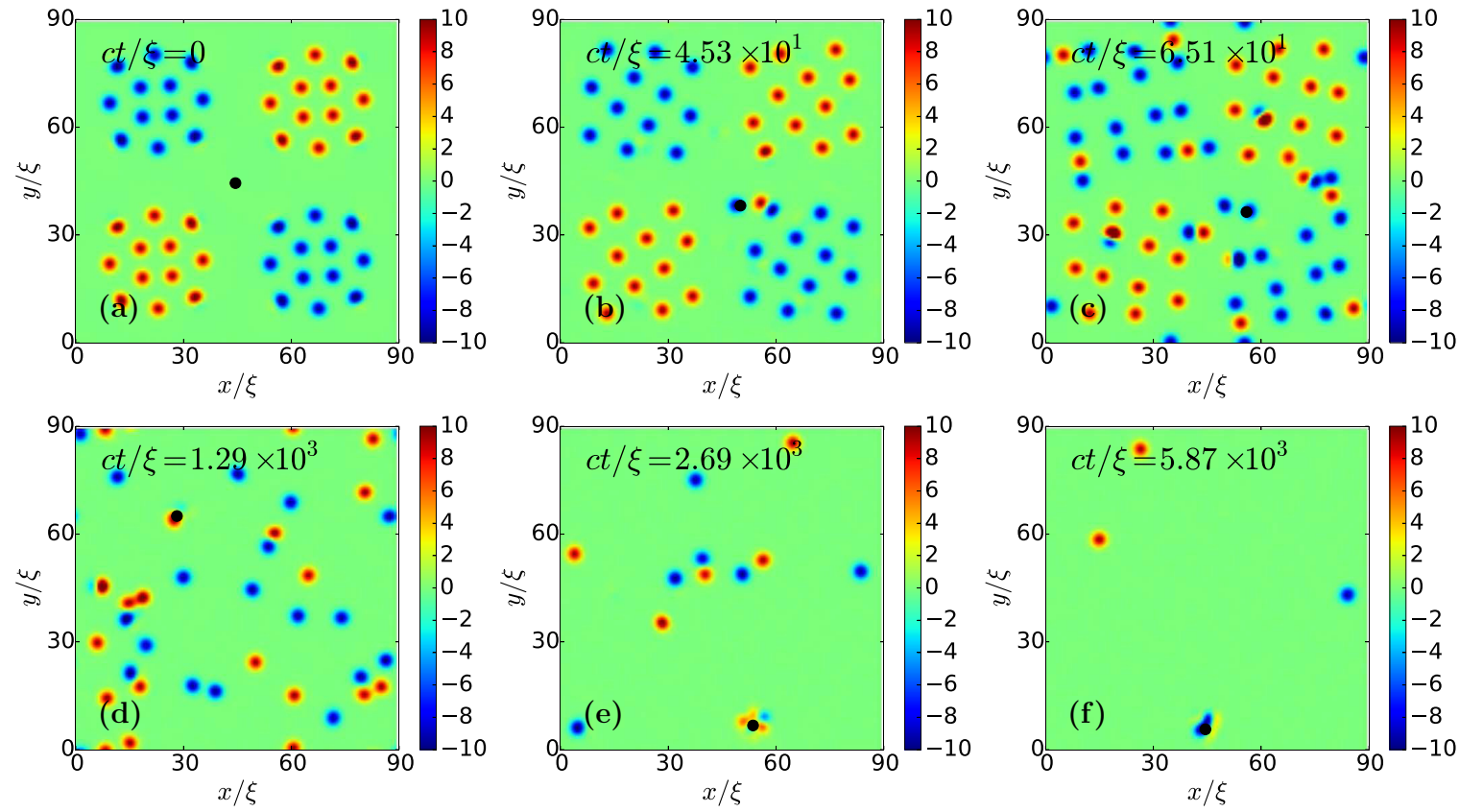

FIG. 11. Spatiotemporal evolution of the filtered vorticity field (derived from the incompressible velocity field), for the neutral particle initially in the presence of counter-rotating vortex clusters (initial configuration ICP3A). The instantaneous position of the particle is shown by a black disk. 


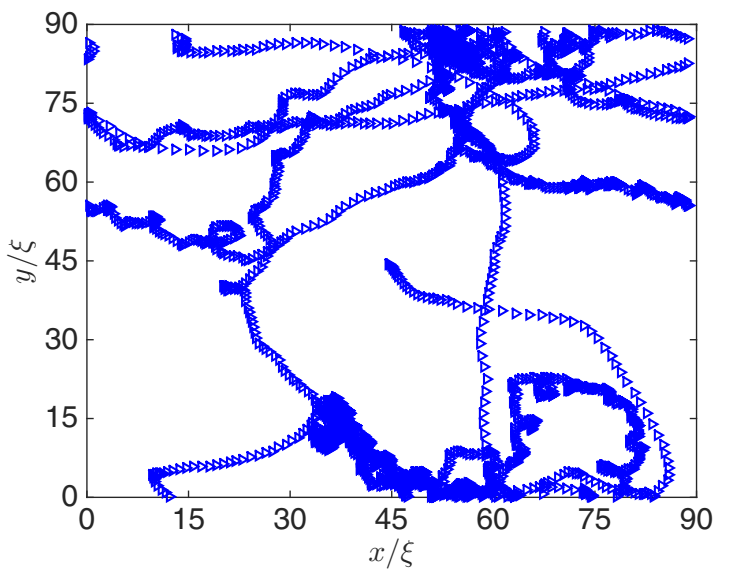

FIG. 12. Trajectory of a neutral particle (denoted by blue triangles), initially in the presence of counter-rotating vortex clusters (initial configuration ICP3A).

illustrative example of many-particle dynamics in the presence of vortices. We generate the initial configuration IPC3B for this purpose in the following three steps. (1) We use the ARGLE to prepare a minimum-energy state with two clusters of positive and negative vortices; each cluster has four vortices of the same sign, and the positive and the negative clusters rotate in opposite directions (for preparation details, see Appendix D2). (2) We prepare a state with four stationary particles Pt : 1 , $\mathrm{Pt}: 2$, Pt $: 3$, and Pt : 4 at the coordinates $(3 \pi / 2 \xi, \pi / 2 \xi)$, $(\pi / 2 \xi, \pi / 2 \xi),(\pi / 2 \xi, 3 \pi / 2 \xi)$, and $(3 \pi / 2 \xi, 3 \pi / 2 \xi)$, respectively, which correspond to the centers of vortex clusters; (3) the states obtained in the steps (1) and (2) are combined together, by multiplying their wave functions, for the initial configuration ICP3B that is then used in the GPE to study the dynamics of this system. Note that to study the particle-field dynamics for this initial configuration we have set the speed of sound $c=2$.

In Figs. 14(a)-14(i) we show the spatiotemporal evolution of the density field $\rho(\mathbf{r}, t)$. In the initial stages of the dynamical evolution of the system, the particles $\mathrm{Pt}: 1-\mathrm{Pt}: 4$ remain sta- tionary at the respective centers of the rotating-vortex clusters, because our initial configuration has fourfold symmetry $\left(C_{4}\right)$ and we prepare it by using the ground state of the vortex clusters. (By contrast, in Sec. IIIC, our initial configuration is not the ground of the vortex clusters.) However, at around $c t / \xi=1.05 \times 10^{3}$, an instability sets in as a result of which the particle Pt : 3 starts to move out; it is then trapped by the negative vortex in front of it [see Fig. 14(c), top-left vortex cluster]; the trapped particle now rotates along with the other three vortices. Similarly, the motion of the other particles also becomes unstable and they are trapped in vortices, in their respective clusters; Fig. 14(d) shows that the particle Pt : 2 is trapped by a vortex at $c t / \xi \simeq 1.13 \times 10^{3}$ and the particles $\mathrm{Pt}: 1$ and $\mathrm{Pt}: 4$ are trapped by vortices at $c t / \xi \simeq 1.20 \times 10^{3}$ [see Fig. 14(e)]. Moreover, at $c t / \xi \simeq 1.31 \times 10^{3}$ the trapped particle $\mathrm{Pt}: 3$ and its vortex form a complex by including another negative vortex of the cluster [see Fig. 14(f), topleft vortex cluster]. In Fig. 15(a) we show the phase of the wave function $\psi$; in such a plot, the vortices are the points around which the phase changes from zero to $2 \pi$; the top-left quadrant still has four vortices, although two on the left are very close by and are held together in a particle-two-vortex complex. The particle-two-vortex complex and the trapped particles continue rotating along with the other vortices, in their respective clusters, over the rest of the simulation time, as we show in Figs. 14(g)-14(i). The Video M11 [21] illustrates the spatiotemporal evolution of the four neutral particles and the density field $\rho(\mathbf{r}, t)$. If the simulation time is further extended, the vortex-particle clusters will expand and result in a complex motion of the particles and vortices, which is akin to $2 \mathrm{D}$ superfluid turbulence.

We plot the trajectories of the four particles Pt : 1 (blue curve), Pt : 2 (red curve), Pt : 3 (green curve), and Pt : 4 (black curve) in Fig. 15(b). These particles move along roughly circular trajectories; however, their motions are not completely periodic in time, so these trajectories meander away from perfectly closed curves and, therefore, fill out two-dimensional areas. This is a signature of either (a) ergodic behavior, e.g., with quasiperiodic temporal evolution or (b) chaotic time evolution. To distinguish between (a) and (b), we examine
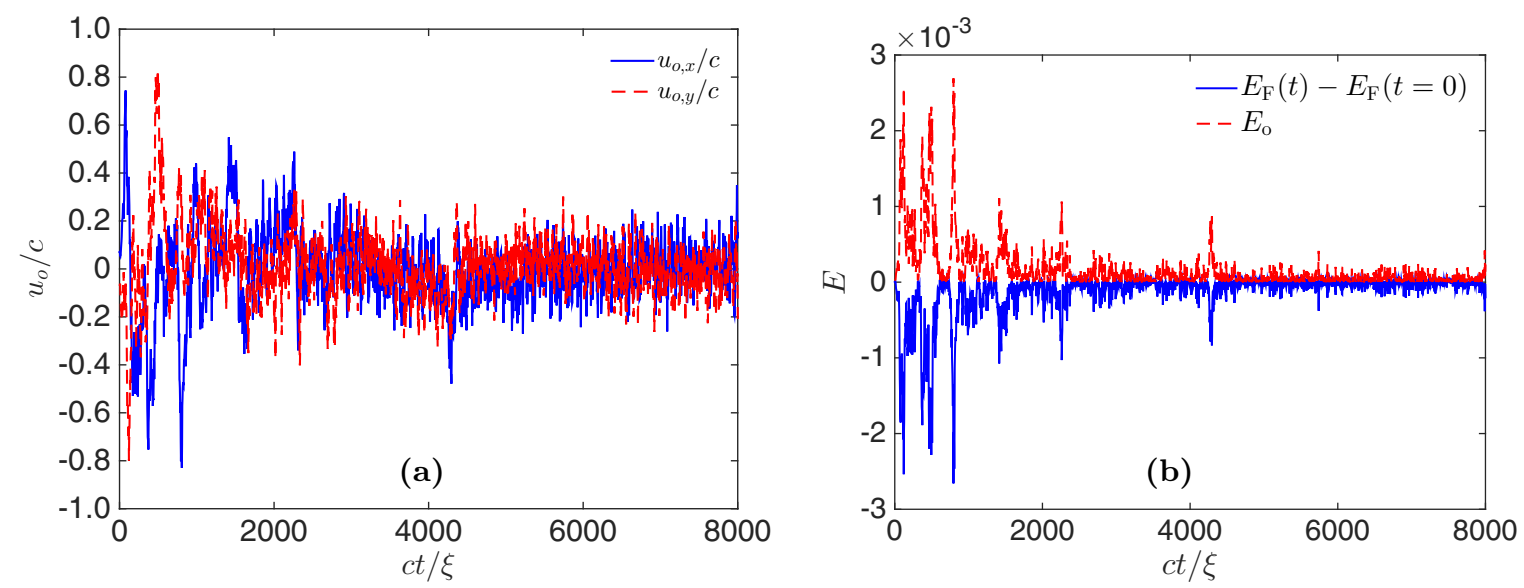

FIG. 13. Plots vs time $t$ of (a) $u_{\mathrm{o}, \mathrm{x}}$ (blue solid curve) and $u_{\mathrm{o}, \mathrm{y}}$ (red dashed curve); (b) $\delta E_{\text {field }}=E_{\mathrm{F}}(t)-E_{\mathrm{F}}(t=0)$ (blue solid curve) and $E_{\mathrm{o}}$ (red dashed curve), obtained from the dynamical evolution of the neutral particle in the presence of counter-rotating vortex clusters (initial configuration ICP3A). 

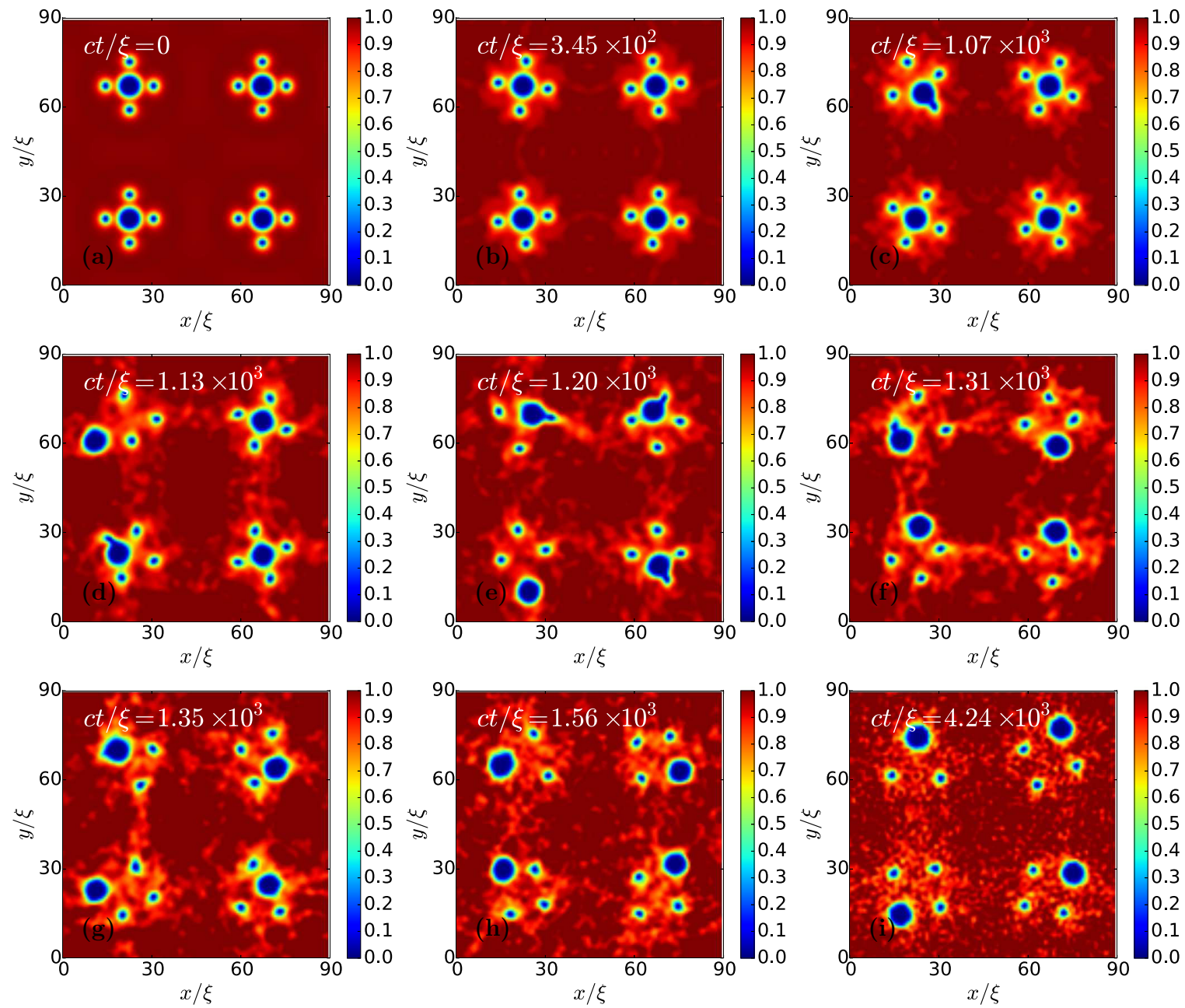

FIG. 14. Spatiotemporal evolution of the density field $\rho(\mathbf{r}, t)$ shown via pseudocolor plots, for the four neutral particles (large blue patches), initially placed at the centers of the counter-rotating vortex clusters (initial configuration ICP3B).

the time series of $u_{\mathrm{o}, \mathrm{x}}$ and $u_{\mathrm{o}, \mathrm{y}}$ for all the four particles in Figs. 16(a)-16(d). The principal peaks in these power spectra can be indexed as $n_{a} \omega_{a}+n_{b} \omega_{b}$, where $n_{a}$ and $n_{b}$ are integers and there are two main incommensurate frequencies $\omega_{a}$ and $\omega_{b}$ (i.e., $\omega_{a} / \omega_{b}$ is an irrational number). For example, in Fig. 17(a) the frequency $\omega_{c}$, of one of the peaks in the power spectrum $S(\omega)$, can be written as $\omega_{c}=2 \omega_{b}-\omega_{a}$ (here $\omega_{c}=$ $0.0 .01777, \omega_{a}=0.01443$, and $\left.\omega_{b}=0.0161\right)$. This labeling of
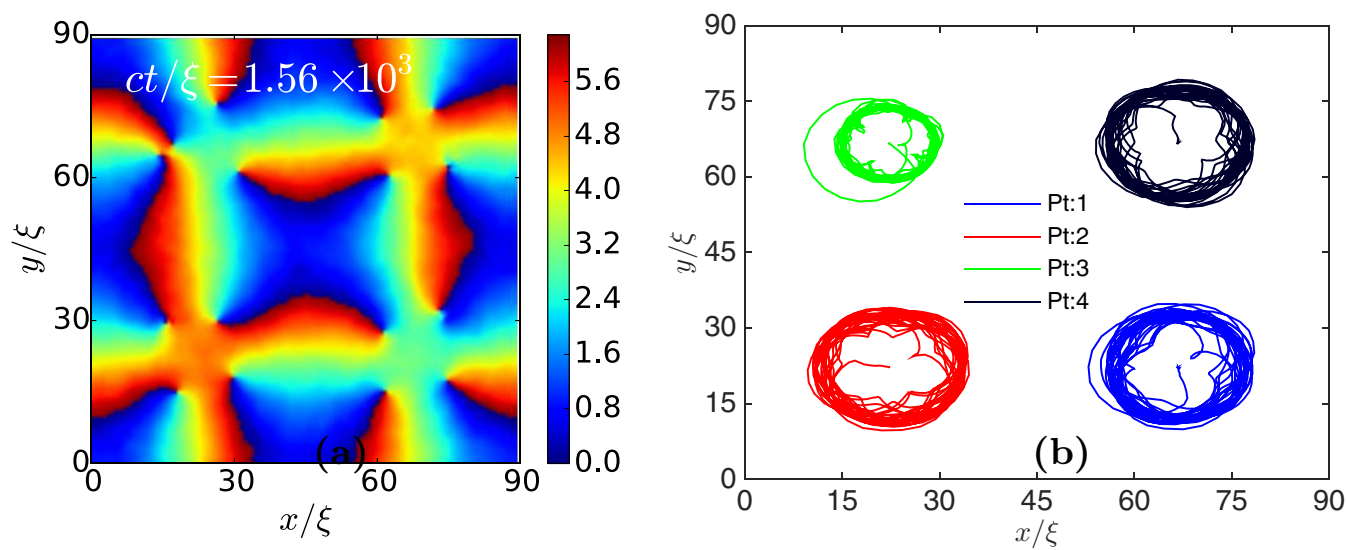

FIG. 15. (a) Pseudocolor plot of the phase of $\psi(\mathbf{x}, t)$; (b) trajectories of four neutral particles Pt : 1 (blue curve, right bottom), Pt : 2 (red curve, left bottom) Pt : 3 (green curve, left top), and Pt : 4 (black curve, right top), in the presence of counter-rotating vortex clusters (initial configuration ICP3B). 

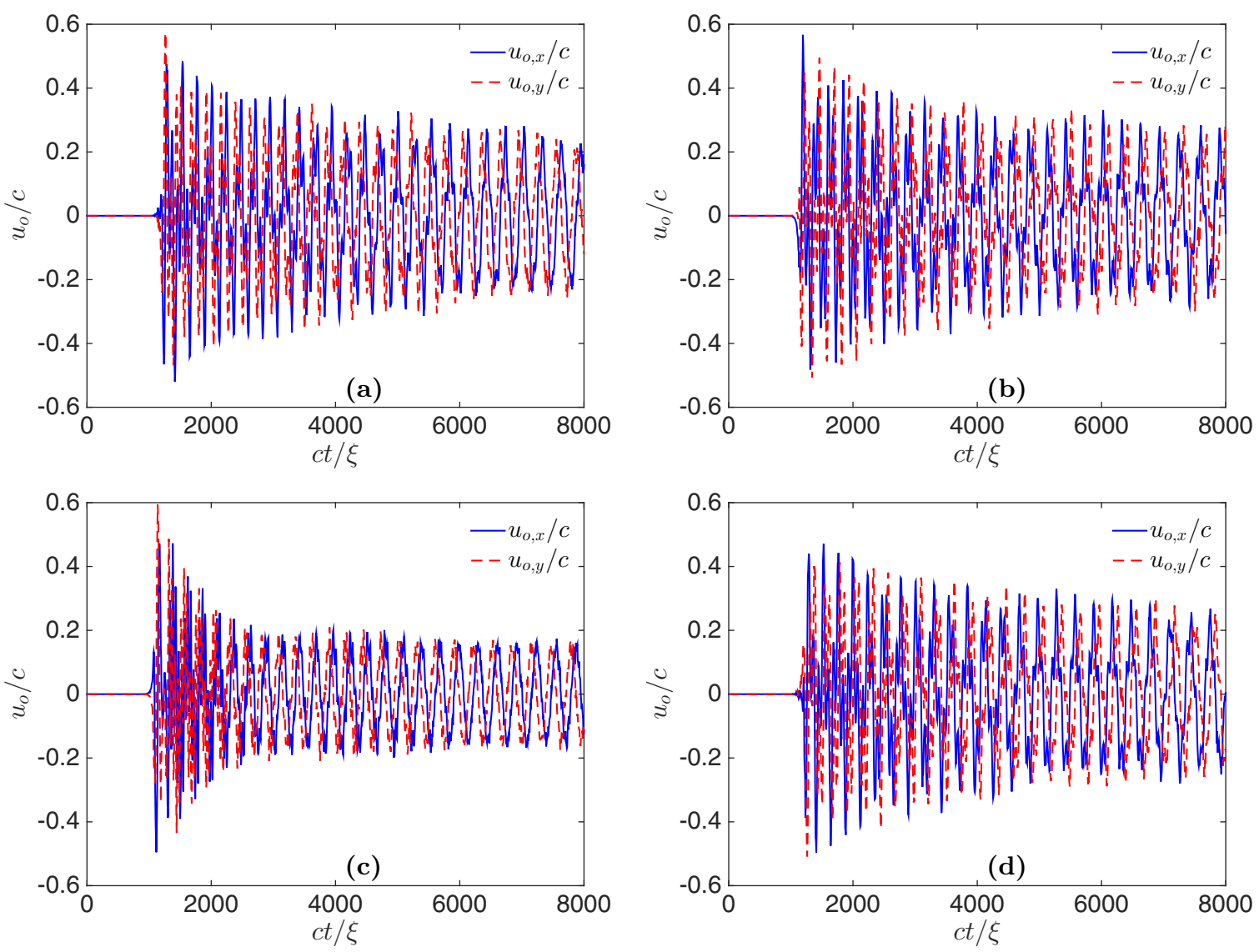

FIG. 16. Plots vs time $t$ of $u_{\mathrm{o}, \mathrm{x}}$ (blue solid curve) and $u_{\mathrm{o}, \mathrm{y}}$ (red dashed curve) for the four neutral particles (a) Pt : 1, (b) Pt : 2, (c) Pt : 3, and (d) Pt : 4, in the presence of counter-rotating vortex clusters (initial configuration ICP3B).

peaks indicates clearly that the temporal evolution of Pt : 1 is quasiperiodic.

\section{CONCLUSIONS}

We have carried out an extensive DNS to investigate the interaction of particles and fields in the 2D GPE, in both simple and turbulent flows. At the one-particle level, we explore, for light, neutral, and heavy particles, the nature of their dynamics in the superfluid, when a constant external force acts on them; in particular, we show, by a careful consideration of the effects of the particle mass, how the motion of such particles can become chaotic. We demonstrate that the interaction of a particle with vortices leads to dynamics that depends sensitively on the particle characteristics. The motion of a single particle through a three-dimensional (3D) GP superfluid has been explored in Refs. [12,13]. Our work complements and extends significantly these earlier studies by (a) considering the 2D GPE, (b) examining the particle-mass dependence of the dynamics, and (c) showing that, after the emission of a vortex-antivortex pair, the temporal evolution of the particle position can be periodic or chaotic.

We extend our studies to assemblies of particles and vortices and demonstrate that their dynamics show rich, turbulent spatiotemporal evolution. In particular, we systematize the spatiotemporal evolution of an initial configuration in which one particle is placed in front of a translating vortex-antivortex pair.
Here our study goes well beyond the recent work presented in Ref. [22], which considers vortex scattering by impurities in a Bose-Einstein condensate; in this study the impurities are static; by contrast, in our work the particle has nontrivial dynamics. Note that, in the limit of large particle mass, our results are akin to those of Ref. [22], with the vortices moving in a superfluid background with a fixed impurity potential. Moreover, our one-particle studies are of direct relevance to the experiments of Refs. [23,24], in which vortex shedding is examined in a BEC with a repulsive Gaussian laser beam that is moved through the condensate. Another system for which our results may prove to be useful is the quantum fluid of light in nonlinear optical systems [25].

Next we examine the complex motions of two particles placed in front of a translating vortex-antivortex pair. An important point that emerges from our study is that, once a particle becomes coincident with a vortex, we can use this particle as a tracer that can track vortex motion. This is of direct experimental relevance; indeed, recent experiments have employed frozen hydrogen particles to track quantized vortices in superfluid helium [2-6].

We have shown that both neutral and light particles can act as tracers for the vortex (i.e., they can be used to track the vortex core). Both of these particles have their limitations for such tracking as is clear from Figs. 6 and 8; the initial response of a neutral particle to the vortex flow is milder than that of a light particle, but the subsequent fluctuations are stronger 

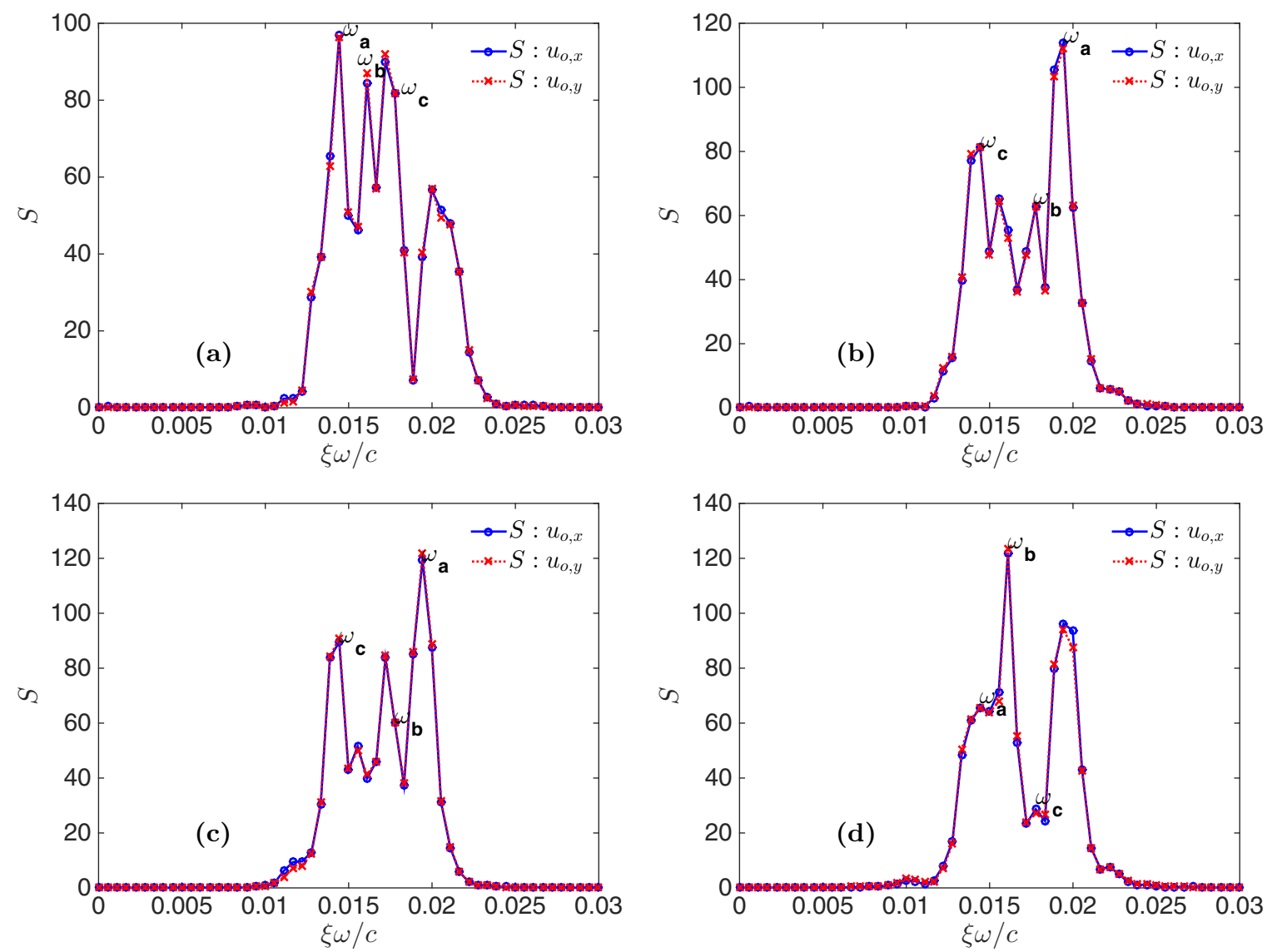

FIG. 17. Plots of power spectra, denoted generically by $S(\omega)$, of the time series of $u_{\mathrm{o}, \mathrm{x}}$ (blue solid curve with circles), $u_{\mathrm{o}, \mathrm{y}}$ (red dotted curve with cross) (see Figs. 16) for the four neutral particles (initial configuration ICP3B) (a) Pt : 1, $\omega_{c}=2 \omega_{b}-\omega_{a}\left(\omega_{a}=0.01443, \omega_{b}=0.0161\right.$, and $\left.\omega_{c}=0.01777\right)$; (b) Pt : 2, $\omega_{c}=3 \omega_{b}-2 \omega_{a}\left(\omega_{a}=0.01943, \omega_{b}=0.01777\right.$, and $\left.\omega_{c}=0.01443\right)$; (c) Pt : 3, $\omega_{c}=3 \omega_{b}-2 \omega_{a}\left(\omega_{a}=0.01943\right.$, $\omega_{b}=0.01777$, and $\left.\omega_{c}=0.01443\right)$; (d) Pt $: 4, \omega_{c}=2 \omega_{b}-\omega_{a}\left(\omega_{a}=0.01443, \omega_{b}=0.0161\right.$, and $\left.\omega_{c}=0.01777\right)$. The frequencies $\omega_{a}, \omega_{b}$, and $\omega_{c}$ that we give are associated with $S(\omega)$ for $u_{\mathrm{o}, \mathrm{x}}$ (blue curves with circles).

for the former. The question of which particle to use for such tracking depends on (a) the system under consideration and (b) to what extent the initial condition can be controlled. Therefore, if the initial perturbation of the flow by a light particle can be ignored, then such a particle should track a vortex core better than neutral or heavy particles. It is worth recalling here that, in classical turbulent flows, light particles are used to track vortex cores.

Finally, our investigations show, for the illustrative examples of (a) a single particle moving in the presence of complex spatial distribution of vortices and (b) four particles in the presence of counter-rotating vortex clusters, how the interactions of particles and fields in a 2D GP superfluid can lead to rich, turbulent, and spatiotemporally chaotic evolution. We hope our work will lead to experimental studies of such spatiotemporal chaos in superfluid helium and BECs.

At the level of our simplified description of a system of vortices and particles in two dimensions, the notions of a point vortex getting trapped on a heavy particle and the neutral or light particle getting trapped on the vortex are intuitively appealing and in consonance with our simple numerical experiments involving initial configurations with a vortex pair and one or two particles. However, the spatiotemporal evolution of the initial configuration with four neutral particles in the presence of counter-rotating vortices is not at all intuitively obvious. In this case, following an instability, the neutral particles get trapped on one of the four vortices of their respective vortex cluster, forming a particle-vortex complex. For one of the four particle-vortex complexes, the subsequent interaction of a vortex with the particle-vortex complex leads to the trapping of this vortex on the latter. Therefore, general statements, based on the mass class, about the spatiotemporal evolution of vortex-and-particle assemblies can be made only in cases with very simple initial configurations; for slightly more complicated configurations, the exact dynamics are strongly influenced by the vortex and particle configurations.

\section{ACKNOWLEDGMENTS}

We thank the Indo-French Centre for Applied Mathematics (IFCAM) for financial support. R.P. and V.S. thank the Council of Scientific and Industrial Research (India), University Grants Commission (India), Department of Science and Technology (India) for support and the Supercomputing Education and Research Centre, IISc, India for computational resources. V.S. acknowledges support from Centre Franco-Indien pour la Promotion de la Recherche Avancée (CEFIPRA) Project No. 4904. V.S. and R.P. thank ENS, Paris for hospitality and 
M.B. thanks IISc, Bangalore for hospitality. We are grateful to G. Krstulovic for useful discussions.

\section{APPENDIX A: NOTE ON UNITS}

The steps given below constitute an easy exercise, however, we give them to avoid any confusion; moreover, this will form a useful background for the Appendixes on initial data preparation. Consider the field part of the full Lagrangian (1),

$$
\begin{aligned}
\mathcal{L}= & \int_{\mathcal{A}}\left[\frac{i \hbar}{2}\left(\psi^{*}(\mathbf{r}, t) \frac{\partial \psi(\mathbf{r}, t)}{\partial t}-\psi(\mathbf{r}, t) \frac{\partial \psi^{*}(\mathbf{r}, t)}{\partial t}\right)\right. \\
& -\frac{\hbar^{2}}{2 m} \nabla \psi(\mathbf{r}, t) \cdot \nabla \psi^{*}(\mathbf{r}, t)+\mu|\psi(\mathbf{r}, t)|^{2}-\frac{g}{2}|\psi(\mathbf{r}, t)|^{4} \\
& \left.-\sum_{i=1}^{\mathcal{N}_{0}} V_{\mathcal{P}}\left(\mathbf{r}-\mathbf{q}_{i}\right)|\psi(\mathbf{r}, t)|^{2}\right] d \mathbf{r},
\end{aligned}
$$

which we rewrite as follows:

$$
\begin{aligned}
\mathcal{L}= & \int_{\mathcal{A}}\left[\frac{i \hbar}{2 m}\left(\tilde{\psi}^{*}(\mathbf{r}, t) \frac{\partial \tilde{\psi}(\mathbf{r}, t)}{\partial t}-\tilde{\psi}(\mathbf{r}, t) \frac{\partial \tilde{\psi}^{*}(\mathbf{r}, t)}{\partial t}\right)\right. \\
& -\frac{\hbar^{2}}{2 m^{2}} \nabla \tilde{\psi}(\mathbf{r}, t) \cdot \nabla \tilde{\psi}^{*}(\mathbf{r}, t)+\frac{\hbar}{m} \frac{\mu}{\hbar}|\tilde{\psi}(\mathbf{r}, t)|^{2} \\
& -\frac{\hbar}{m} \frac{g}{2 \hbar m}|\tilde{\psi}(\mathbf{r}, t)|^{4} \\
& \left.-\sum_{i=1}^{\mathcal{N}_{0}} \frac{\hbar}{m} \frac{V_{\mathcal{P}}\left(\mathbf{r}-\mathbf{q}_{i}\right)}{\hbar}|\tilde{\psi}(\mathbf{r}, t)|^{2}\right] d \mathbf{r} \\
= & \int_{\mathcal{A}}\left[i \alpha_{0}\left(\tilde{\psi} \tilde{\psi}^{*}(\mathbf{r}, t) \frac{\partial \tilde{\psi}(\mathbf{r}, t)}{\partial t}-\tilde{\psi}(\mathbf{r}, t) \frac{\partial \tilde{\psi} \tilde{r}^{*}(\mathbf{r}, t)}{\partial t}\right)\right. \\
& -2 \alpha_{0}^{2} \nabla \tilde{\psi}(\mathbf{r}, t) \cdot \nabla \tilde{\psi}^{*}(\mathbf{r}, t)+2 \alpha_{0} \tilde{\mu}|\tilde{\psi}(\mathbf{r}, t)|^{2} \\
& -2 \alpha_{0} \frac{\tilde{g}}{2}|\tilde{\psi}(\mathbf{r}, t)|^{4} \\
& \left.-\sum_{i=1}^{\mathcal{N}_{0}} 2 \alpha_{0} \tilde{V}_{\mathcal{P}}\left(\mathbf{r}-\mathbf{q}_{i}\right)|\tilde{\psi}(\mathbf{r}, t)|^{2}\right] d \mathbf{r} .
\end{aligned}
$$

From the above steps it follows that $\tilde{g}=g / \hbar m, \tilde{\mu}=\mu / \hbar$, $\tilde{V}_{\mathcal{P}}=V_{\mathcal{P}} / \hbar$, and $|\tilde{\psi}|^{2}=m|\psi|^{2}$; thus $|\tilde{\psi}|^{2}$ can be directly interpreted as the mass density of the superfluid. From the Lagrangian Eq. (A3) we obtain the following equation of motion for the field $\tilde{\psi}$ :

$$
2 \alpha_{0} i \frac{\partial \tilde{\psi}}{\partial t}=2 \alpha_{0}\left[-\alpha_{0} \nabla^{2}+\tilde{g}|\tilde{\psi}|^{2}-\tilde{\mu}+\tilde{V}_{\mathcal{P}}\right] \tilde{\psi} .
$$

Hereafter we drop the ${ }^{\sim}$ symbol for notational convenience in the discussions of Appendixes B-D.

\section{APPENDIX B: ADVECTIVE REAL GINZBURG-LANDAU EQUATION (ARGLE)}

Gross-Pitaevskii hydrodynamics is compressible, so, when vortices are present, we get dynamics that is dominated by acoustic waves. To minimize these acoustic emissions, it is useful to prepare initial conditions by using the advective-realGinzburg-Landau equation (ARGLE) given in Refs. [26,27].
The initial states we want emerge as the large-time-asymptotic solutions of the following ARGLE:

$$
\frac{\partial \psi}{\partial t}=\alpha_{0} \nabla^{2} \psi-g|\psi|^{2} \psi+\mu \psi-i \mathbf{u}_{\mathrm{adv}} \cdot \nabla \psi-\frac{\mathbf{u}_{\mathrm{adv}}^{2}}{4 \alpha_{0}} \psi
$$

These states minimize the free-energy functional

$$
\begin{aligned}
\mathcal{F}_{\mathrm{ARGLE}}\left(\psi, \psi^{*}\right)= & \int d^{3} x\left(\alpha_{0}\left|\nabla \psi-i \frac{\mathbf{u}_{\mathrm{adv}}}{2 \alpha_{0}} \psi\right|^{2}\right. \\
& \left.+\frac{1}{2} g|\psi|^{4}-\mu|\psi|^{2}\right) .
\end{aligned}
$$

Here $\mathbf{u}_{\mathrm{adv}}$ is the imposed flow velocity. In Eqs. (B1) and (B2) a potential term can be included to prepare initial states with particles.

\section{APPENDIX C: NUMERICAL IMPLEMENTATION OF ARGLE}

For time stepping in the ARGLE we use the following implicit-Euler method:

$$
\psi(t+\Delta t)=\frac{\psi(t)+N L(t) \Delta t}{1-L \Delta t},
$$

where we suppress the spatial argument of $\psi, L=\alpha_{0} \nabla^{2}$, and $N L=\left(\mu-g|\psi|^{2}\right) \psi-i \mathbf{u}_{\mathrm{adv}} \cdot \nabla \psi-\frac{\mathbf{u}_{\mathrm{adv}}^{2}}{4 \alpha_{0}} \psi$. The field $\psi$ at the time step $(n+1)$ is given by

$$
\hat{\psi}_{n+1}=\frac{\hat{\psi}_{n}+\Delta t\left(\mu-g \mid \widehat{\left.\psi_{n}\right|^{2} \psi_{n}}-i \widehat{\mathbf{u}_{\mathrm{adv}} \cdot \nabla} \psi_{n}-\frac{\widehat{\mathbf{u}_{\mathrm{dv}}^{2}} \psi_{n}}{4 \alpha_{0}}\right)}{1-\left(-\alpha_{0} k^{2}\right) \Delta t} .
$$

We use the Newton method to find the stable and the unstable fixed points of the above equation; this is equivalent to finding $\psi_{*}$, such that

$$
F\left(\psi_{*}\right) \equiv \psi_{*}(t)-\psi_{*}(t+\Delta t)=0 .
$$

Every Newton step requires the solution, for $\delta \psi$, of

$$
\frac{\delta F}{\delta \psi} \delta \psi=-F(\psi)
$$

We obtain this by an iterative, biconjugate-gradient-stabilized method (BiCGSTAB) [28]. This method uses $[\delta F / \delta \psi]$ over an arbitrary field $\phi$ :

$$
\begin{aligned}
\frac{\delta F}{\delta \psi} \phi= & \frac{-\Delta t}{1-L \Delta t}\left[L \phi+g\left(2|\psi|^{2} \phi+\psi^{2} \phi^{*}\right)-i \mathbf{u}_{\mathrm{adv}} \cdot \nabla \phi\right. \\
& \left.-\left(\mathbf{u}_{\mathrm{adv}}^{2} / 4 \alpha_{0}\right) \phi\right] .
\end{aligned}
$$

\section{APPENDIX D: PREPARATION OF INITIAL STATES WITH VORTICES}

\section{Preparation of a translating vortex-antivortex pair: $\psi_{\text {pair }}$}

We give below the steps required to prepare $\psi_{\text {pair }}$ (see Refs. [26,27]):

(1) Initialize $\psi(x, y)=\exp (i x)$ for $l_{\min }<y<l_{\max }$ and $\psi(x, y)=1$ otherwise.

(2) Evolve $\psi$ by using ARGLE, with $u_{\text {adv }}=0$, and allow the vortex-antivortex pair that is generated to contract until it reaches the desired value of the pair length $d$. 

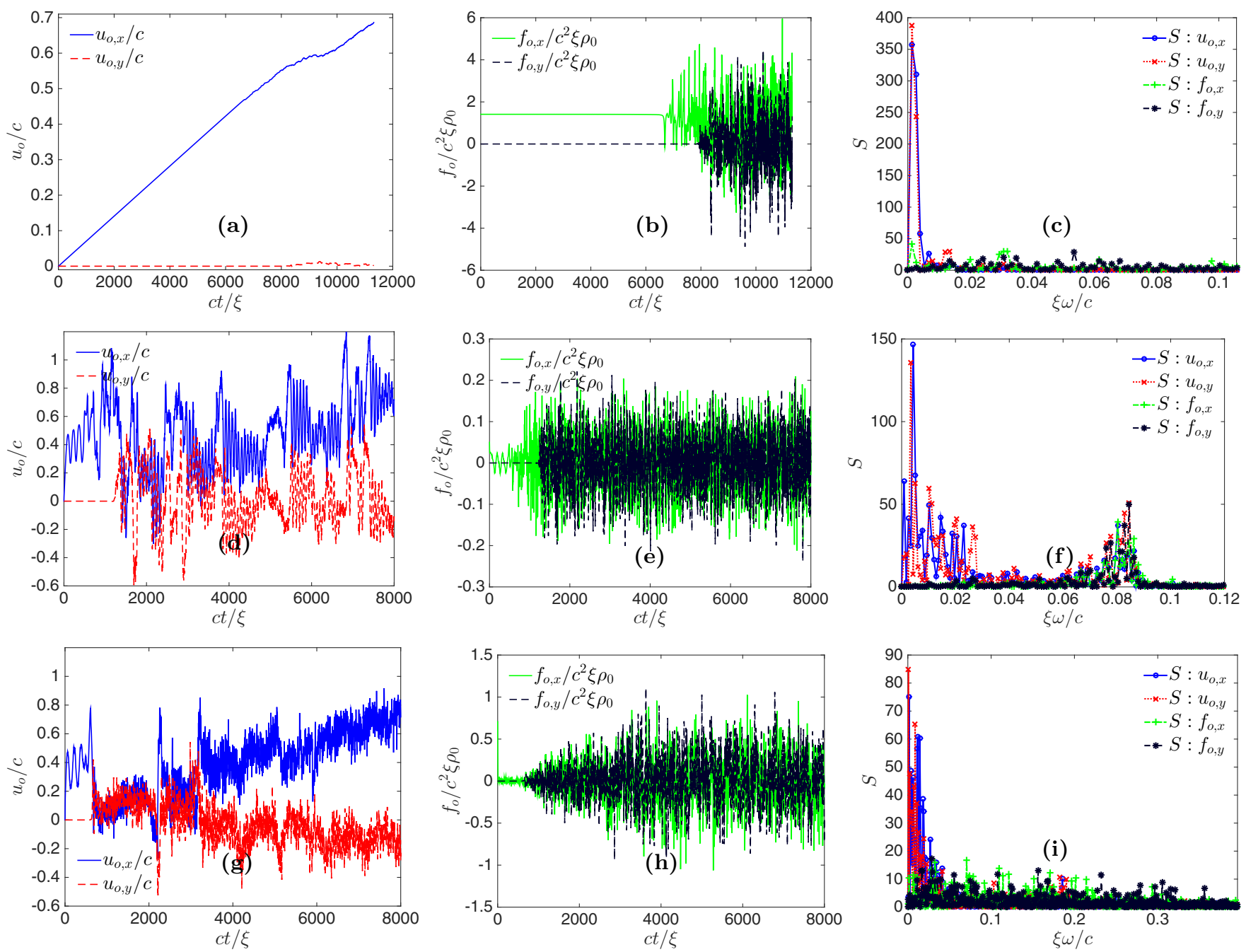

FIG. 18. Plots of the Cartesian components of (a) velocity $u_{\mathrm{o}, \mathrm{x}}$ and $u_{\mathrm{o}, \mathrm{y}}$, (b) force $f_{\mathrm{o}, \mathrm{x}}$ and $f_{\mathrm{o}, \mathrm{y}}$, and (c) power spectra of the quantities time series in (a) and (b), for the heavy particle $\left(\mathcal{M}=374, \mathbf{F}_{\text {ext }}=1.42 c^{2} \xi \rho_{0} \hat{\mathbf{x}}\right)$. Plots in (d)-(f) and (g)-(i) are the analogs of plots in (a)-(c), for the neutral $\left(\mathcal{M}=1, \mathbf{F}_{\text {ext }}=0.71 c^{2} \xi \rho_{0} \hat{\mathbf{x}}\right)$ and light $\left(\mathcal{M}=0.0374, \mathbf{F}_{\text {ext }}=0.71 c^{2} \xi \rho_{0} \hat{\mathbf{x}}\right)$ particles, respectively. Power spectra, denoted generically by $S(\omega)$, of the time series of $u_{\mathrm{o}, \mathrm{x}}, u_{\mathrm{o}, \mathrm{y}}, f_{\mathrm{o}, \mathrm{x}}$, and $f_{\mathrm{o}, \mathrm{y}}$ are plotted vs the angular frequency $\omega$ for the above three cases.

(3) Evolve $\psi$, from the previous step, by using ARGLE, with $\mathbf{u}_{\mathrm{adv}}=u \hat{x}$, so that the contraction of the vortex-antivortex pair stops.

(4) Use Newton's method, coupled with BiCGSTAB, to find the exact state of the vortex-antivortex pair for $\mathbf{u}_{\mathrm{adv}}$ in step (3) above. This Newton method helps to speed up the convergence to the desired solution [the solution is a saddle point of Eq. (B1); the ARGLE procedure, if used alone, first converges, but finally ends up diverging].

\section{Preparation of counter-rotating vortex clusters}

The steps involved in the preparation of $\psi_{\text {cluster }}$ are outlined below:

(1) Initialize $\psi_{e}\left(\lambda_{1}, \lambda_{2}\right)=\frac{\left(\lambda_{1}+\iota \lambda_{2}\right)}{A} \tanh \left(\frac{A}{\sqrt{2} \xi}\right)$, where $\lambda_{1}=$ $\sqrt{2} \cos x, \lambda_{2}=\sqrt{2} \cos y$, and $A=\sqrt{\lambda_{1}^{2}+\lambda_{2}^{2}}$.

(2) Prepare $\psi_{4}=\psi_{e}\left(\lambda_{1}-\eta, \lambda_{2}\right) \psi_{e}\left(\lambda_{1}, \lambda_{2}-\eta\right) \psi_{e}\left(\lambda_{1}+\right.$ $\left.\eta, \lambda_{2}\right) \psi_{e}\left(\lambda_{1}, \lambda_{2}+\eta\right)$, where $\eta=1 / \sqrt{2}$.
(3) Prepare $\psi_{\text {cluster }}=\left(\psi_{4}\right)^{\left[\gamma_{d} / 4\right]}$, where $\gamma_{d}=8 /\left(4 \pi \alpha_{0}\right)$ and $[\cdot]$ denotes the integer part of a real number.

(4) Evolve $\psi_{\text {cluster }}$ by using ARGLE with $u_{\mathrm{adv}, \mathrm{x}}=$ $\sin (x) \cos (y), u_{\mathrm{adv}, \mathrm{y}}=-\cos (x) \sin (y)$ to minimize acoustic emission.

For more details on the preparation of a counter-rotating vortex cluster, see Ref. [26].

\section{APPENDIX E: ADDITIONAL FIGURES}

In this Appendix we give additional plots to compliment the discussion of Secs. III A and III B.

In Fig. 18 we show plots similar to those in Fig. 2 of Sec. III A, but for a higher value of the amplitude of the external force acting on the particle. This enhances the chaotic nature of the particle dynamics; in particular, the power spectra of the Cartesian components of $u_{\mathrm{o}}$ and $f_{\mathrm{o}}$ [see Figs. 18(c), 18(f), and 18(i)] have a broad range of frequencies for all three types of particles. 

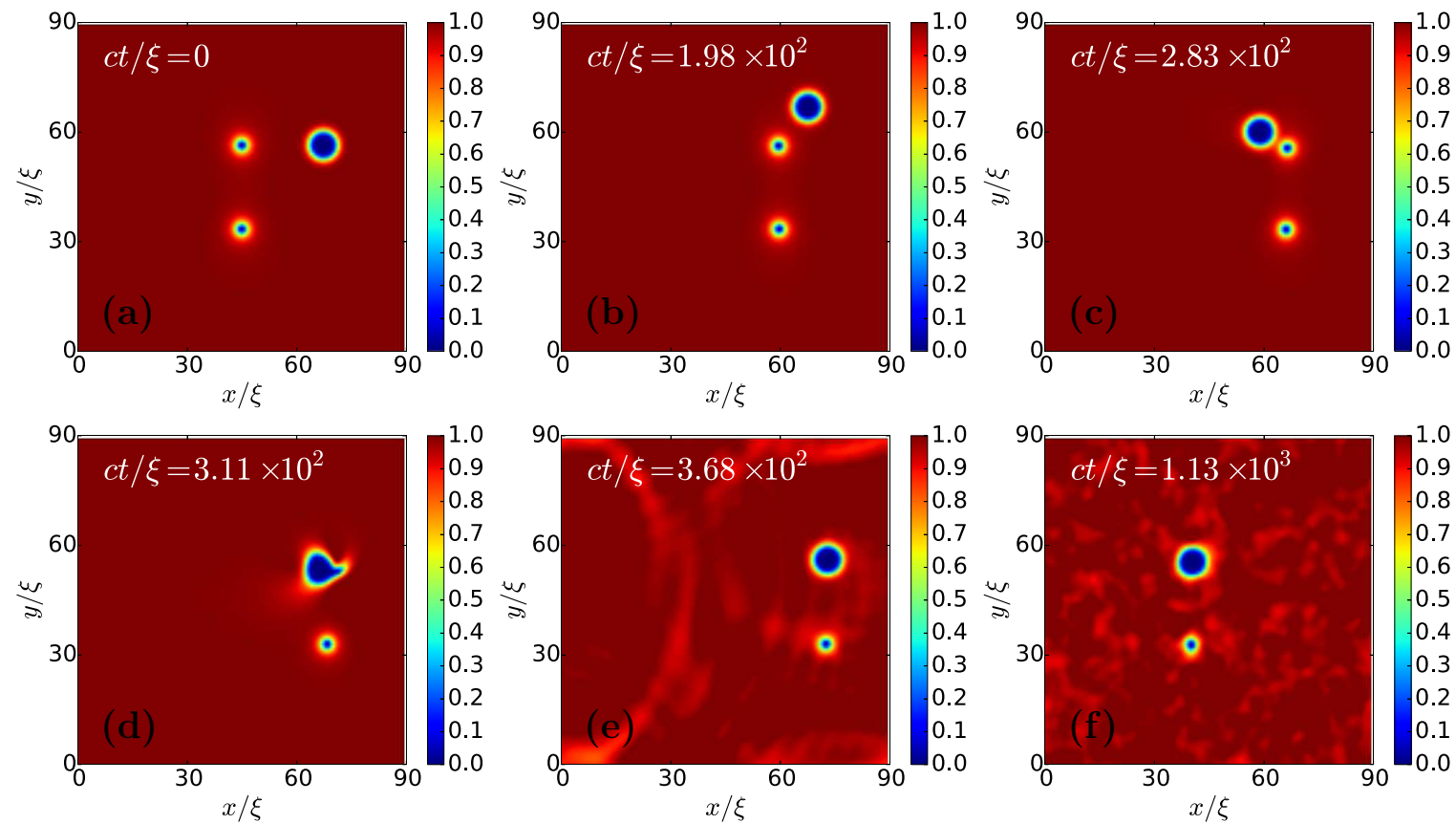

FIG. 19. Light particle interacting with a translating vortex-antivortex pair. Spatiotemporal evolution of the density field $\rho(\mathbf{r}, t)$ shown via pseudocolor plots, for a light particle placed in the path of the positive (upper) vortex of a translating vortex-antivortex pair (initial configuration ICP2A).

The light particle is more sensitive to the flow around a vortex than the neutral particle. Figure 19 shows the pseudocolor plots of the density field at different instants of time for a light particle placed in front of a trans- lating vortex-antivortex pair. The response of the light particle is very dramatic: while moving around the positive vortex, it is pushed almost to the back of the vortex.
[1] R. J. Donnelly, Quantized Vortices in Helium II, Vol. 2 (Cambridge University Press, Cambridge, UK, 1991).

[2] G. P. Bewley, D. P. Lathrop, and K. R. Sreenivasan, Nature (London) 441, 588 (2006).

[3] G. P. Bewley, M. S. Paoletti, K. R. Sreenivasan, and D. P. Lathrop, Proc. Natl. Acad. Sci. USA 105, 13707 (2008).

[4] G. P. Bewley, K. R. Sreenivasan, and D. P. Lathrop, Exp. Fluids 44, 887 (2008).

[5] D. E. Zmeev, F. Pakpour, P. M. Walmsley, A. I. Golov, W. Guo, D. N. McKinsey, G. G. Ihas, P. V. E. McClintock, S. N. Fisher, and W. F. Vinen, Phys. Rev. Lett. 110, 175303 (2013).

[6] M. La Mantia and L. Skrbek, Phys. Rev. B 90, 014519 (2014).

[7] N. Spethmann, F. Kindermann, S. John, C. Weber, D. Meschede, and A. Widera, Phys. Rev. Lett. 109, 235301 (2012).

[8] T. Frisch, Y. Pomeau, and S. Rica, Phys. Rev. Lett. 69, 1644 (1992).

[9] C. Nore, C. Huepe, and M. E. Brachet, Phys. Rev. Lett. 84, 2191 (2000).

[10] C. Huepe and M.-E. Brachet, Physica D 140, 126 (2000).

[11] C.-T. Pham, C. Nore, and M.-É. Brachet, Physica D 210, 203 (2005).

[12] T. Winiecki, B. Jackson, J. F. McCann, and C. S. Adams, J. Phys. B: At., Mol., Opt. Phys. 33, 4069 (2000).

[13] T. Winiecki and C. S. Adams, Europhys. Lett. 52, 257 (2000).

[14] G. E. Astrakharchik and L. P. Pitaevskii, Phys. Rev. A 70, 013608 (2004).
[15] E. Varga, C. F. Barenghi, Y. A. Sergeev, and L. Skrbek, J. Low Temp. Phys. 183, 215 (2016).

[16] V. Shukla, M. Brachet, and R. Pandit, Phys. Rev. A 94, 041602 (2016).

[17] R. Pandit, D. Banerjee, A. Bhatnagar, M. Brachet, A. Gupta, D. Mitra, N. Pal, P. Perlekar, S. S. Ray, V. Shukla, and D. Vincenzi, Phys. Fluids 29, 111112 (2017).

[18] V. Shukla, M. Brachet, and R. Pandit, New J. Phys. 15, 113025 (2013).

[19] http://www.fftw.org.

[20] G. Krstulovic and M. Brachet, Phys. Rev. E 83, 066311 (2011).

[21] See Supplemental Material at http://link.aps.org/supplemental/ 10.1103/PhysRevA.97.013627 for the videos and additional figures.

[22] A. Griffin, G. W. Stagg, N. P. Proukakis, and C. F. Barenghi, J. Phys. B 50, 115003 (2017).

[23] T. W. Neely, E. C. Samson, A. S. Bradley, M. J. Davis, and B. P. Anderson, Phys. Rev. Lett. 104, 160401 (2010).

[24] W. J. Kwon, G. Moon, S. W. Seo, and Y. Shin, Phys. Rev. A 91, 053615 (2015).

[25] I. Carusotto and C. Ciuti, Rev. Mod. Phys. 85, 299 (2013).

[26] C. Nore, M. Abid, and M. E. Brachet, Phys. Fluids 9, 2644 (1997).

[27] V. Shukla, M. Brachet, and R. Pandit, arXiv:1412.0706.

[28] H. A. Van der Vorst, SIAM J. Sci. Stat. Comput. 13, 631 (1992). 